\title{
Detailed Characterization of Laser-Produced Astrophysically-Relevant Jets Formed via a Poloidal Magnetic Nozzle
}

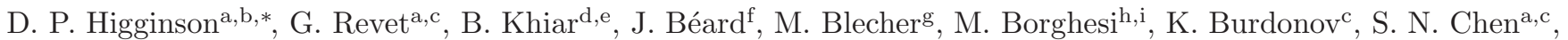 \\ E. Filippov ${ }^{\mathrm{j}, \mathrm{k}}$, D. Khaghani ${ }^{\mathrm{l}}$, K. Naughton ${ }^{\mathrm{h}}$, H. Pépin $^{\mathrm{m}}$, S. Pikuz ${ }^{\mathrm{j}, \mathrm{k}}$, O. Portugall ${ }^{\mathrm{f}}$, C. Riconda ${ }^{\mathrm{n}}$, R. Riquier ${ }^{\mathrm{a}, \mathrm{o}}$,
}

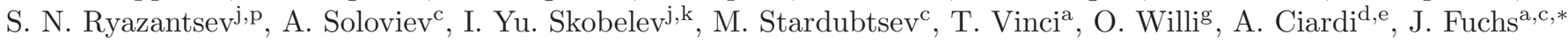

${ }^{a}$ LULI - CNRS, École Polytechnique, CEA: Université Paris-Saclay; UPMC Univ Paris 06: Sorbonne Universités - F-91128 Palaiseau \author{
cedex, France \\ ${ }^{b}$ Lawrence Livermore National Laboratory, Livermore, California 94550, USA \\ ${ }^{c}$ Institute of Applied Physics, 46 Ulyanov Street, 603950 Nizhny Novgorod, Russia \\ ${ }^{d}$ Sorbonne Universités, UPMC Univ. Paris 6, UMR 8112, LERMA, F-75005, Paris, France \\ ${ }^{e}$ LERMA, Observatoire de Paris, PSL Research University, CNRS, UMR 8112, F-75014, Paris France \\ ${ }^{f} L N C M I, U P R$ 3228, CNRS-UGA-UPS-INSA, 31400 Toulouse, France \\ ${ }^{g}$ Institut für Laser-und Plasmaphysik, Heinrich-Heine-Universität Düsseldorf, D-40225 Düsseldorf, Germany \\ ${ }^{h}$ The Queen's University of Belfast, Belfast BT7 1 NN, United Kingdom \\ ${ }^{i}$ Institute of Physics of the ASCR, ELI-Beamlines project, Na Slovance 2, 18221 Prague, Czech Republic \\ ${ }^{j}$ Joint Institute for High Temperatures, RAS, 125412, Moscow, Russia \\ ${ }^{k}$ National Research Nuclear University 'MEPhI', 115409 Moscow, Russia \\ ${ }^{l}$ GSI Helmholtzzentrum für Schwerionenforschung GmbH, 64291 Darmstadt, Germany \\ ${ }^{m} I N R S$-ÉMT, 1650 bd. L. Boulet, J3X1S2 Varennes, Québec, Canada \\ ${ }^{n}$ LULI, Sorbonne Universités-UPMC Univ. Paris 06, École Polytechnique, CNRS, CEA, 75005 Paris, France \\ ${ }^{\circ} C E A, D A M$, DIF, 91297 Arpajon, France \\ ${ }^{p}$ M. V. Lomonosov Moscow State University, Moscow 119991, Russia
}

\begin{abstract}
The collimation of astrophysically-relevant plasma ejecta in the form of narrow jets via a poloidal magnetic field is studied experimentally by irradiating a target situated in a $20 \mathrm{~T}$ axial magnetic field with a $40 \mathrm{~J}, 0.6 \mathrm{~ns}, 0.7 \mathrm{~mm}$ diameter, high-power laser. The dynamics of the plasma shaping by the magnetic field are studied over 70 ns and up to 20 mm from the source by diagnosing the electron density, temperature and optical self-emission. These show that the initial expansion of the plasma is highly magnetized, which leads to the formation of a cavity structure when the kinetic plasma pressure compresses the magnetic field resulting in an oblique shock [A. Ciardi et al., Phys. Rev. Lett. 110, 025002 (2013)]. The resulting poloidal magnetic nozzle generates a standing conical shock that collimates the plasma into a narrow jet [B. Albertazzi et al., Science 346, 325 (2014).]. At distances far from the target, the jet is only marginally magnetized and maintains a high aspect ratio due to its high Mach-number $(\mathbb{M} \sim 20)$ and not due to external magnetic pressure. The formation of the jet is evaluated over a range of laser intensities $\left(10^{12}-10^{13} \mathrm{~W} / \mathrm{cm}^{2}\right)$, target materials and orientations of the magnetic field. Plasma cavity formation is observed in all cases and the viability of long-range jet formation is found to be dependent on the orientation of the magnetic field.
\end{abstract}

\section{Introduction}

\subsection{Recent Work and Astrophysical Implications}

Recent laboratory studies[1, 2, 3] and pertinent astrophysical simulations $[4,5,6]$ have shown the viability of poloidal (i.e. axial) magnetic fields to directly result in the collimation of wide-angle outflows and the formation of jets in astrophysical accreting systems [7, 8], such as young stellar objects (YSO). In particular, this mechanism has been shown to generate large aspect ratio (length:diameter $>1: 10)$ jets and through the formation of a long-standing

\footnotetext{
* Corresponding authors

Email addresses: higginson2@llnl.gov (D. P. Higginson), julien.fuchs@polytechnique.edu (J. Fuchs)
}

and relatively stationary conical shock is suggested to be at the origin of long-time x-ray emission observed from such objects (e.g. HH 154) [9, 10, 11].

The dynamics of the laboratory-produced jets was shown to be scalable to YSOs jets[1,2] as both systems are to a first approximation well described by magnetohydrodynamics (MHD) [12, 13]. The laboratory evidence for the poloidal collimation of jets thus offers an explanation of the observed long range collimation of young stellar jets[14]. We note that this mechanism is complementary to magneto-centrifugally launched disk winds[15] (i.e. self-collimation) which explain the launching of wide-angle outflows and their collimation into jets, as well as the removal of angular momentum from accretion disks; a mechanism that has been studied both via simulations[16] and 
experiments [17, 18, 19]. Shown below in Eq. (1) is the radial Lorentz force exerted on an ideal MHD plasma in cylindrical coordinates. The force associated with selfcollimation in magneto-cetrifugal models is due to the toroidal magnetic field $B_{\phi}$. On the other hand, the poloidal collimation mechanism that is explored in this paper is due to the presence of an initially axial magnetic field $B_{z}$.

$$
F_{r}=\underbrace{-j_{z} B_{\phi}}_{\text {self-collimation }}+\underbrace{j_{\phi} B_{z}}_{\text {poloidal collimation }}
$$

The process of poloidal magnetic collimation of a laserablated plasma is illustrated in Fig. 1. Without a strong poloidal B-field, the plasma is heated to high temperature and expands into vacuum in all directions, creating a quasi-hemispherical expansion (Fig. 1a). When a strong B-field is applied, as shown in Fig. 1b, the plasma is restricted from expanding radially by the field and expands only until reaching an equilibrium between the total plasma kinetic pressure and magnetic pressure. This causes a conical shock to form and the plasma is subsequently redirected onto the radial axis. As the plasma converges on axis a conical jet is formed and the plasma is collimated into a high Mach-number, high aspect-ratio jet.

The focus of this paper is to robustly characterize these laboratory-generated jets produced by laser-matter interaction and collimated via external axial magnetic fields that we have recently investigated $[1,2]$, as well as to highlight their stability over long temporal duration and under a variety of plasma conditions. Of particular interest is the conclusion that the plasma is only marginally magnetized at distances far away from the target. This indicates that the high aspect ratio of the jet is due mainly to the collimation at the base and then to the high Mach-number Lagrangian ballistic expansion of the flow at large distances. In other words, at large distances from the target, the presence of the magnetic field is unimportant to the collimation of the flow. To make this point clear, we characterize this collimation mechanism as a poloidal magnetic nozzle (PMN) in order to highlight that the collimation mechanism is active near the base of the jet. We note here that the B-fields are called poloidal due to the toroidal symmetry of a jet ejected from an accreting system. In the experimental setup, cylindrical symmetry is apparent and the fields are referred to as axial. Since the system size is very large in the poloidal astrophysical case, these two descriptions are equivalent near the base of the jet.

In Section 2, we explain the experimental configuration of the laser, the Helmholtz coil that drives an external magnetic field, and the suite of experimental diagnostics for observing the electron density (2D-space, time), optical self-emission (1D-space, time) and electron temperature (1D-space). In Section 3 we characterize the plasma based on measurements in different regions and we estimate related plasma parameters in order to give a context to the plasma confinement and jet formation. Note that the char-
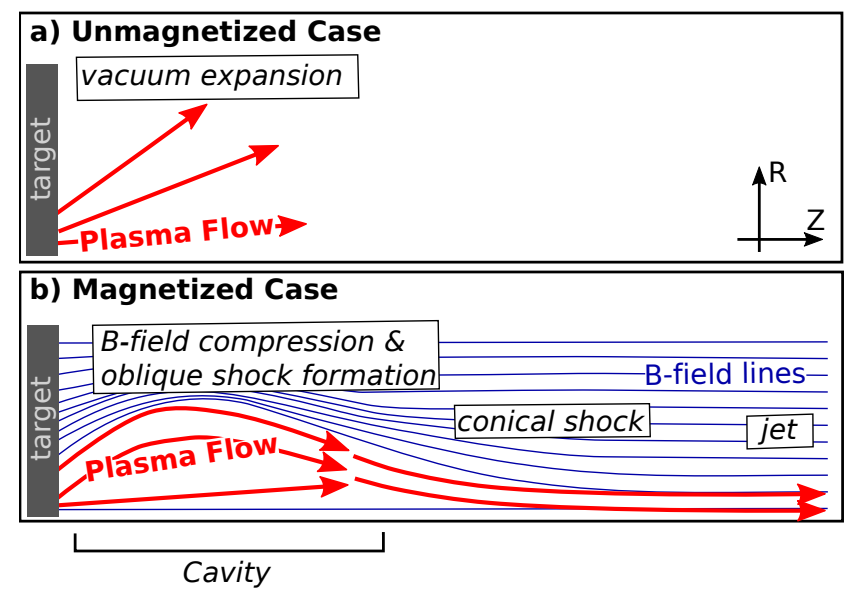

Figure 1: Schematic of plasma expansion into vacuum following the laser-irradiation of the front (right) side of the target. a) Without a strong B-field, the plasma expands in a wide-angle flow. b) With a strong poloidal B-field, the plasma is confined laterally by the Bfield, forms an oblique conical shock and is redirected onto the radial axis. The on-axis conical shock collimates the flow into a jet.

acterization of the dynamics of laser-produced plasma expansion into vacuum has been an active subject of research for over 50 years. Thus, this (unmagnetized) expansion will not be the focus of the present paper, rather we will focus on how such a plasma is shaped into a narrow jet through its interaction with the poloidal B-field.

In Section 4, we describe the formation and evolution of the jet over $23 \mathrm{~mm}$ in space and over $70 \mathrm{~ns}$ in time when the B-field is applied. We detail the formation of a cavity near the target caused by the generation of an oblique shock along the plasma expansion front, which leads to a strong conical shock and the subsequent formation of a long-aspect-ratio jet. In Section 5, we examine the fidelity of the jets to a variety of experimental parameters: we vary the kinetic energy of the expansion by changing the laser energy incident on the target, we vary the atomic composition of the jet by varying the target material and we vary the magnetic field orientation by tilting the target by $45^{\circ}$. We observe that varying the laser energy and target material leads to quantitative differences in the jet formation, but shows similar overall behavior in that the plasma is confined radially and a jet is formed. When the magnetic field is tilted by $45^{\circ}$, the plasma is still confined radially, but the formation of a long-range, narrow jet is not observed.

\subsection{Historical Context}

Investigating the possibility of influencing and guiding the hydrodynamics of high-temperature laser-produced plasmas has been the subject of many investigations, including some very early studies[20, 21] . Most experiments investigated plasma dynamics across B-field, revealing a fraction of the plasma to be confined while another part can move across the B-field via an $E \times B$ drift allowed by the development of polarization E-field in the plasma [21, 22, 23, 24]. 
The growth of flute-like instabilities affecting the plasma dynamics at intermediate levels of magnetization was also demonstrated[25, 26]. We should note however that all these studies were conducted in a regime where only the electrons in the plasma were magnetized, the ions being not or weakly magnetized.

Strong plasma magnetization of laser-produced plasmas has become possible only recently with the development of adequate systems, e.g. at the Laboratory for Laser Energetics[27, 28, 29] (Rochester, NY, USA) and at the Institute of Laser Engineering[30] (Osaka, Japan). However, the B-fields they develop have short spatial ( $\mathrm{mm})$ and temporal (10-100 ns) scales. The platform[31, 32] developed in collaboration between the LNCMI and LULI laboratories (France) lifts these limitations by allowing magnetization of laser produced plasmas up to $40 \mathrm{~T}$ over much larger $(>\mathrm{cm})$ and longer $(>\mu \mathrm{s})$ scales. Such homogeneous and stationary field generation is a key factor in allowing the present observations as it ensures that a homogeneous magnetic field exists over several $\mathrm{cm}$. This field is obtained through the use of large-scale pulsed coils, similarly as what had been done earlier at the Institute of Plasma Physics and Laser Microfusion [33] (Warsaw, Poland) and at the Lawrence Livermore National Laboratory[34] (Livermore, CA, USA), but here we use larger scales and higherstrength B-field so to induce stronger plasma magnetization. Importantly, with such a system, plasma magnetization can be arbitrarily varied in magnitude or direction. Another significant advantage of this system is that it is not explosive and hence debris-free.

With this system, we recently showed that by strongly magnetizing a laser-ablated plasma along its expansion axis, we could produce for the first time long, stable, narrow and dense plasma jets without any supporting media[2] This allowed us to test the validity of a proposed theoretical shaping mechanism of astrophysical narrow jets. The $20 \mathrm{~T}$ B-field could significantly influence the plasma dynamics since the plasma is strongly dominated by the applied B-field at distances greater than $\sim 1 \mathrm{~mm}$ from the target. This is possible since the, order $100 \mathrm{~T}$, selfgenerated B-fields (e.g. Biermann battery, $\nabla n_{e} \times \nabla T_{e}$ ) do not influence the plasma anymore as they are confined to high strength $[35,36]$ at $<0.5 \mathrm{~mm}$ from the target surface, i.e. over very short scales compared to the typical plasma expansion scale. In Ref. [36] it is clear that the Nerst effect confines the strong self-generated B-fields against the target so that the expanding plasma plume has only low-strength B-fields (i.e. $<1 \mathrm{~T}$ ) that should be negligible compared to the applied (20 T) B-field.

We note that many other plasma configurations have been used in the laboratory to investigate jet formation and dynamics[37]: spheromak-like jets[38, 39], pulsed power pinches $[17,18,19]$ and (un-magnetized) laser-plasmas[40, $41,42,43,44]$.
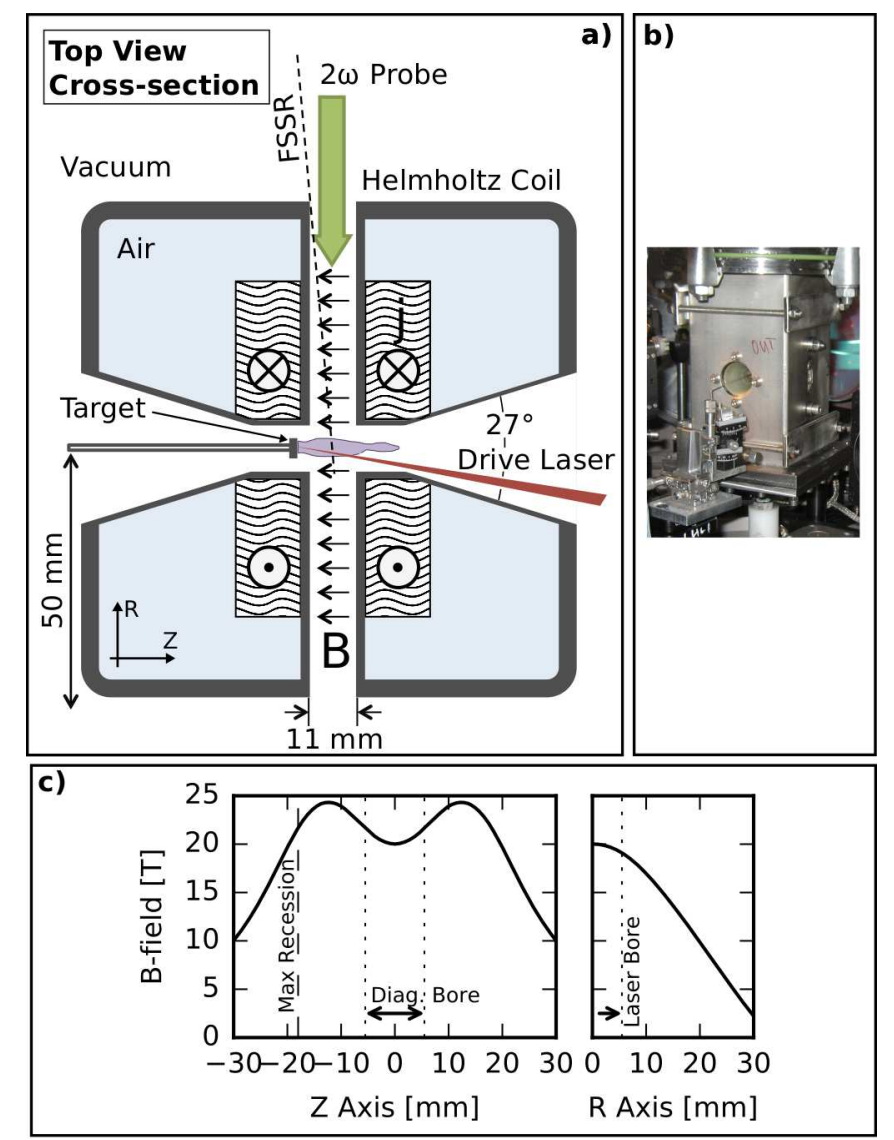

Figure 2: a) Top view schematic of the Helmholtz coil system. The "laser bore" (left-right axis) of the coil would allow a maximum of $27^{\circ}$ full-angle beam (f/2.1) for a laser beam at normal incidence. The "diagnostic bore" (up-down axis) is of constant $11 \mathrm{~mm}$ diameter. b) Photograph of the Helmholtz coil in the chamber looking into the "laser-bore". Motorized stages are not shown. c) Magnetic field profiles along the coil central axes. The target is recessed along the longitudinal (laser bore) axis which is parallel to the field lines. Dotted and dashed lines show the extent of the coil bore and the maximum distance over which the target was recessed.

\section{Experimental Setup}

\subsection{Laser and Target Parameters}

The experiment was performed at the ELFIE laser facility $[45]$ at the Laboratoire pour l'Utilisation des Lasers Intenses (LULI) in France. A schematic of the target and laser setup within the coil is shown in Figure 2. This system employs a Ti:Sa $(\lambda=1057 \mathrm{~nm})$ amplification chain that normally stretches, amplifies and then recompresses via the chirped pulse amplification[46] technique to subpicosecond duration. However, in our experiment we use the beam prior to recompression resulting in a frequency chirped $40 \mathrm{~J}, 0.6 \mathrm{~ns}$ pulse-length beam. The laser was focused through a $2.2 \mathrm{~m}$ focal length lens (f/21) and a random phase plate[47] to achieve a $0.7 \mathrm{~mm}$ diameter focal spot on target. At the maximum energy of $40 \mathrm{~J}$ this achieved an on-target intensity of $2 \times 10^{13} \mathrm{~W} / \mathrm{cm}^{2}$. The laser was incident at $10^{\circ}$ on the front surface of a thick, $2 \mathrm{~mm}$ diameter $\mathrm{CF}_{2}$ (Teflon) disc mounted on a glass stalk 
connected to an encoded motorized stage. The target surface was aligned perpendicular to the magnetic field with a $\pm 5^{\circ}$ uncertainty.

\subsection{Helmholtz Coil and Pulsed Power Driver}

Fig. 2a shows a schematic of the pulsed-power driven Helmholtz coil developed at the Laboratoire National des Champs Magnétiques Intenses (LNCMI)[48] in Toulouse. This device allows for a peak field strength of $40 \mathrm{~T}$, though in practice it was used at $20 \mathrm{~T}$ to increase the lifetime of the coils. This design is similar to the one described in Refs. [31, 2] with the largest difference being increased bore sizes for diagnostic and laser access. The coil and windings are described in Ref. [49] The windings and, thus the current flow, is kept in air to avoid breakdown in the fiber-glass structure. Fig. 2b shows a photograph of the coil in the target chamber. The coil was supported from the top by vacuum tubes and a bellows that allowed the tilt to be adjusted to align the magnetic field with the chamber.

The coils were driven by a $16 \mathrm{kV}, 250 \mu \mathrm{F}, 32 \mathrm{~kJ}$ pulsed power unit[31] developed by the Dresden High Magnetic Field Lab (HLD) at Helmholtz-Zentrum Dresden-Rossendorf (HZDR). The rise time of the coil was $190 \mu \mathrm{s}$, meaning that the magnetic field is constant over the experiment timescale $(<0.1 \mu \mathrm{s})$. As shown in Fig. 2c, the coil was designed to have a large volume of constant magnetic field. In order to probe the jet at distances longer than the size of the bore of the coil, the target was recessed to a precise position inside the coil using the motorized target stages. The magnetic pressure of $20 \mathrm{~T}$ is $160 \mathrm{MPa}$ and the magnetic energy in the center bore $\left(r=5.5 \mathrm{~mm}, V=\frac{4}{3} \pi r^{3}\right)$ is $56 \mathrm{~J}$. This is enough to fully contain the $40 \mathrm{~J}$ laser pulse considering that not all of the laser energy is absorbed and that some of the absorbed energy is lost to non-adiabatic channels (e.g. radiation, ionization).

\subsection{Interferometry}

Interferometry is accomplished via the Mach-Zehnder technique with a frequency doubled probe laser with a pulse length of $5 \mathrm{ps}$ and $\sim 100 \mathrm{~mJ}$ of energy. Figure 3 shows the probe beam trajectory after it has been compressed, frequency doubled and delayed with respect to the nanosecond irradiation beam. To increase the amount of data taken per shot, the probe beam was split in two via a polarizing prism. A delay of 11 ns was added to one of the beams and it was then re-injected back into the original beam path so that both beams were exactly co-aligned. At the end of the beam path, the beams were again separated using a polarizing prism so that each beam was displayed independently on a CCD camera. A halfwave plate was used to divide the beam evenly between the two polarizations. The beams were imaged through a $1.2 \mathrm{~m}$ lens onto an Andor CCD cameras (13x13 $\mu \mathrm{m}$ pixels, 1024x1024 pixel chip size). This setup had a magnification of $1.2 \mathrm{x}$, a $10.8 \mu \mathrm{m}$ resolution and a field of view

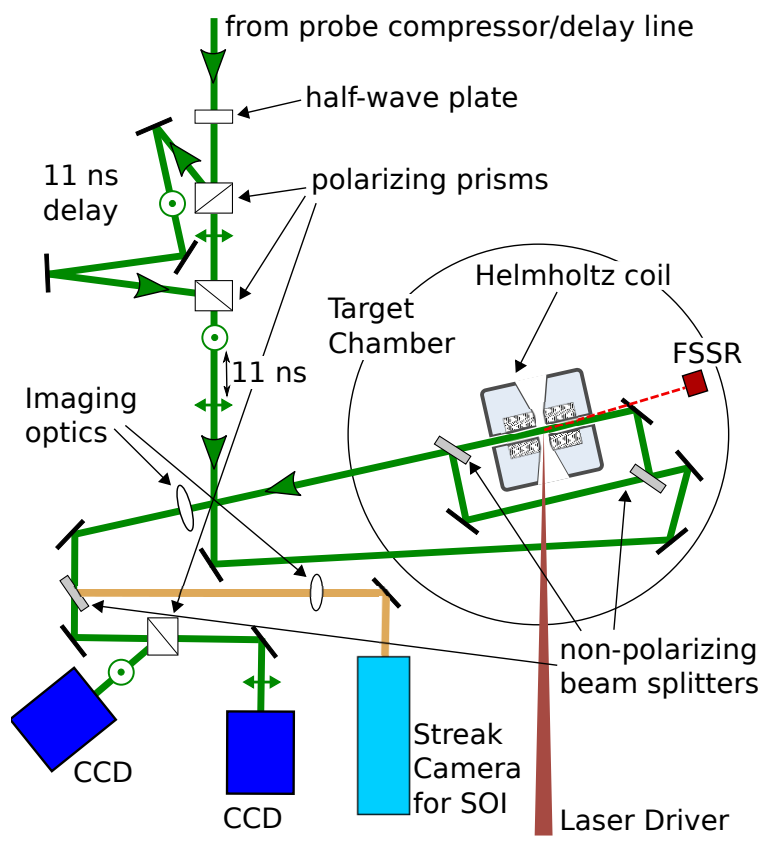

Figure 3: Diagnostic diagram of the experimental setup. The probe beamline is split and recombined so that one beam is delayed by $11 \mathrm{~ns}$. The SOI imaging line looks along an axis identical to the probe beam. The line-of-sight to the FSSR crystal used for x-ray spectroscopy is also shown.

$\sim 11 \mathrm{~mm}$ to capture the entire coil window. The interference fringes were fit with a wavelet model and unwrapped using Neutrino[50]. An Abel inversion[51] was applied to the (background-subtracted) phase to determine the electron density.

\subsection{Streaked Optical Imager (SOI)}

A streaked optical imager (SOI) was used to create a time-resolved 1D image of the plasma self-emission along the jet propagation axis and was centered on the middle of the laser spot. As shown in Fig. 3, this diagnostic used the same optical pathway as the probe beam and thus imaged the plasma from the same angle as the interferometry. After the $1.2 \mathrm{~m}$ lens of the interferometry system, the SOI pathway was split using a non-polarizing beamsplitter and then re-imaged through a $300 \mathrm{~mm}$ focal length lens on to a Hamamatsu C7700 streak camera with an S20 photocathode (sensitive to 200-850 nm wavelengths). Narrowbandwidth (notch) optical filtering was used to block the light from the probe and driver beams. The spatial variation in the cathode efficiency was taken into account using a flat-field calibration[52].

\subsection{X-ray Focusing Spectrometer with Spatial Resolution (FSSR)}

A focusing spectrometer with spatial resolution (FSSR) was used to record the temporally-integrated x-ray emission spectra of the plasma. The diagnostic was set up to resolve x-ray energies in the range of $750-950 \mathrm{eV}$ in the first order of mica crystal reflection and $1500-1900 \mathrm{eV}$ in the 
second order. The diagnostic was aligned along nearly the same line-of-sight as the interferometer with a slight angle in the upwards $\left(\sim 5^{\circ}\right)$ and lateral $\left(\sim 2^{\circ}\right)$ directions (i.e. small enough to neglect skewing). The relative intensities of He-like spectral lines (e.g. $\mathrm{He}_{\beta}, \mathrm{He}_{\gamma}, \mathrm{He}_{\delta}$ ) radiated by transitions in the Fluorine ions were analyzed. The ratios between multiple pairs of lines is used to constrain both the electron density $n_{e}$ and temperature $T_{e}$. Since this diagnostic is not temporally resolved the values are intrinsically weighted towards times of highest emissivity, which is a function of both temperature and density. A detailed methodology of this analysis technique is presented in Ref. $[53,54]$.

\section{Summary of Plasma and Magnetization Param- eters}

To provide a context of these magnetized jets, we will now consider the conditions of the plasma at various regions of interest using the experimentally evaluated temperatures, densities and inferred flow velocities of the plasma. These conditions are presented in Table 1. The experimental data from which we take these values will be presented in detail in Section 4. We now define the different regions that we will evaluate and then we will derive the plasma parameters presented in Table 1 . For each region we define a time $t$ and distance of interest $z$; this allows us to estimate the flow velocity $u_{d}=z / t$ following a Lagrangian ballistic expansion model where all of the acceleration occurs at the time of the laser irradiation at $t=0$, a model based on previous simulations[1] of the flow velocity in the same configuration.

\section{Region 1: Initial Expansion}

Region 1 considers the very initial expansion of the plasma near the target. In the first frame of the interferometry images shown in Fig. 4a/5a we see that the fastest portion of the plasma that can be detected by our diagnostic has expanded to $\sim 6 \mathrm{~mm}$ and has an electron density of $n_{e}=1 \times 10^{17} \mathrm{~cm}^{-3}$ at a time of $6 \mathrm{~ns}$. From this we conclude that this portion of the plasma is traveling at $u_{d}=1000 \mathrm{~km} / \mathrm{s}$. There may be faster portions of the plasma that are not observable to our diagnostic, but these will of lower density and should not play a major role in the shaping of the jet. Since we expect that the plasma will reach the hottest temperatures at this initial expansion time, we use the hottest temperature measured with the FSSR, $T_{e}=80 \mathrm{eV}$ (shown in Fig. 10) for this region. We note that as the FSSR is time-integrated so this temperature may be an underestimate.

\section{Region 2: Cavity}

Region 2 defines the cavity region described in Fig. 1b that can be observed in Fig. 5b. The cavity will be examined at two distinct periods in time and space. We first define Region $2 \mathrm{a}$, as the period when the cavity is expanding, here we take the location as $2 \mathrm{~mm}$ and the time as
$16 \mathrm{~ns}$, giving $u_{d}=120 \mathrm{~km} / \mathrm{s}$. The Region $2 \mathrm{~b}$ we define as the period when the cavity is contracting/contracted, where the location is $1 \mathrm{~mm}$ and the time is $23 \mathrm{~ns}$, which gives $u_{d}=45 \mathrm{~km} / \mathrm{s}$ for the plasma that flows there are this stage. The FSSR shows $T_{e} \simeq 20 \mathrm{eV}$ and $T_{e} \simeq 35 \mathrm{eV}$ and the interferometry yields $n_{e} \simeq 3 \times 10^{18} \mathrm{~cm}^{-3}$ and $n_{e} \simeq 5 \times 10^{18} \mathrm{~cm}^{-3}$ for Region $2 \mathrm{a}$ and $2 \mathrm{~b}$, respectively.

\section{Region 3: Propagating Jet}

Region 3 represents the collimated jet that propagates with very high aspect ratio over long distances (see Fig. 1b, Fig. 5e). We know that the jet, at is observable density of $10^{18} \mathrm{~cm}^{-3}$, has reached at least a distance of $22 \mathrm{~mm}$ from the target $60 \mathrm{~ns}$ from the laser ablation from which we infer a bulk velocity of $330 \mathrm{~km} / \mathrm{s}$. In this region we are limited by the observed range of our diagnostic of $22 \mathrm{~mm}$, however we expect that the range of the jet is much longer, with a higher Mach number and larger aspect ratio than we capture experimentally. In this region we determine $T_{e}=10 \mathrm{eV}$ and $n_{e}=1 \times 10^{18} \mathrm{~cm}^{-3}$ from the FSSR (this is an upper estimate and extrapolation from the data) and interferometer, respectively.

\subsection{Thermodynamic Quantities}

To determine the average charge state $\bar{Z}$ of the plasma, we used tabular data from FLYCHK[55]. We find that these values shift less than $10 \%$ for density variations of even $10 \mathrm{x}$ around a density of $10^{18} \mathrm{~cm}^{-3}$. We assume a quasi-neutral plasma $\left(n_{e}=\bar{Z}_{i} n_{i}\right)$, where the average charge state $\bar{Z}_{i}=\frac{2}{3} \bar{Z}_{F}+\frac{1}{3} \bar{Z}_{C}$ is the stoichiometric average of the two species. The mass density is found in the same manner, $\rho_{i}=n_{i} m_{i}=n_{i}\left(2 m_{F}+m_{C}\right) / 3$.

To get an idea of the relevant velocities in our system we look at the sound and thermal speeds of the plasma. We assume that $T_{i}=T_{e}$, an adiabatic index $\gamma=5 / 3$, with stoichiometric percentages $S_{\alpha}$, masses $m_{\alpha}$ and charge states $Z_{\alpha}$ of the individual ions $\alpha$. This gives an effective sound speed $C_{s}=\left(\gamma T_{e} \sum S_{\alpha} Z_{\alpha} m_{\alpha}^{-1}\right)^{1 / 2}$ and the thermal velocities $v_{T \alpha}=\left(T_{\alpha} / m_{\alpha}\right)^{1 / 2}$ of the species $\alpha$. We define the ion quantities as the stoichiometric average of the two species (i.e. $v_{T i}=\frac{2}{3} v_{T F}+\frac{1}{3} v_{T C}$ ). The Alfvén speed is $v_{A}=B / \sqrt{\mu_{0} \rho_{i}}$, where $B$ is the magnetic field and $\mu_{0}$ is the vacuum permeability. The Mach number $\mathbb{M}$ and the Alfvénic Mach number $\mathbb{M}_{A}$ are defined as the ratio of the bulk velocity to the sound and Alfvén speeds, respectively.

\subsection{Particle Motion}

We calculate the gyroradius $r_{L \alpha}=v_{T \alpha} / \omega_{c \alpha}$ of the species $\alpha$, where $\omega_{c \alpha}=Z_{\alpha} q_{e} B / m_{\alpha}$ is the gyrofrequency. For a $20 \mathrm{~T}$ B-field, $\omega_{c e}^{-1}=3 \times 10^{-4} \mathrm{~ns} / \mathrm{rad}$ and $\omega_{c i}^{-1}=$ $1.4 \mathrm{~ns} / \mathrm{rad}$. Note that these frequencies should be roughly similar for all of the different regions considering that the gyrofrequency is only dependent on the strength of the magnetic field and, in the case of ions, the charge state. For these calculations we assume a constant value of $20 \mathrm{~T}$ for the field and charge states of $\bar{Z}_{C}=4$ and $\bar{Z}_{F}=7$ are used and stoichiometrically averaged. 


\begin{tabular}{c|c|c|c|c|l} 
& 1 & $2 \mathrm{a}$ & $2 \mathrm{~b}$ & 3 & \\
\hline$z$ & 6 & 2 & 1 & 22 & $\mathrm{~mm}$ \\
$t$ & 6 & 16 & 23 & 60 & $\mathrm{~ns}$
\end{tabular}

Measurements

\begin{tabular}{l|c|c|c|c|l}
$n_{e}$ & $1.10^{17}$ & $3.10^{18}$ & $5.10^{18}$ & $1.10^{18}$ & $\mathrm{~cm}^{-3}$ \\
$T_{\mathrm{e}}$ & 80 & 20 & 35 & 10 & $\mathrm{eV}$ \\
$u_{d}$ & 1000 & 120 & 43 & 370 & $\mathrm{~km} / \mathrm{s}$
\end{tabular}

Avg. Charge States

\begin{tabular}{l|c|c|c|c|c}
$\bar{Z}_{\mathrm{F}}$ & 7 & 5 & 6 & 4 & \\
$\bar{Z}_{\mathrm{C}}$ & 5 & 4 & 4 & 4 & \\
$\bar{Z}_{i}$ & 6.3 & 4.7 & 5.3 & 4 & \\
Ion Densities \\
$n_{i}$ & $2.10^{16}$ & $6.10^{17}$ & $9.10^{17}$ & $2.10^{17}$ & $\mathrm{~cm}^{-3}$ \\
$\rho_{i}$ & $4.10^{-7}$ & $2.10^{-5}$ & $3.10^{-5}$ & $7.10^{-6}$ & $\mathrm{~g} / \mathrm{cm}^{3}$
\end{tabular}

\section{Velocities}

\begin{tabular}{l|c|c|c|c|c}
$C_{s}$ & 70 & 30 & 43 & 20 & $\mathrm{~km} / \mathrm{s}$ \\
$v_{T e}$ & 3800 & 1900 & 2500 & 1300 & $\mathrm{~km} / \mathrm{s}$ \\
$v_{T i}$ & 22 & 11 & 14 & 7.7 & $\mathrm{~km} / \mathrm{s}$ \\
$v_{A}$ & 850 & 130 & 110 & 210 & $\mathrm{~km} / \mathrm{s}$
\end{tabular}

\section{Length Scales}

\begin{tabular}{l|c|c|c|c|c}
$r_{L i}$ & 30 & 20 & 70 & 760 & $\mu \mathrm{m}$ \\
$\lambda_{i}$ & 5.3 & $3.10^{-2}$ & $3.10^{-2}$ & $3.10^{-2}$ & $\mu \mathrm{m}$ \\
$r_{L e}$ & 1.1 & 0.5 & 0.7 & 0.4 & $\mu \mathrm{m}$ \\
$\lambda_{e}$ & 150 & 0.4 & 0.7 & 0.4 & $\mu \mathrm{m}$
\end{tabular}

Time Scales

\begin{tabular}{l|c|c|c|c|c}
$\tau_{a d}$ & 1 & 8 & 23 & 2.7 & $\mathrm{~ns}$ \\
$\tau_{h e}$ & 0.6 & 480 & 220 & 790 & $\mathrm{~ns}$ \\
$\tau_{m}$ & 75 & 13 & 26 & 5.3 & $\mathrm{~ns}$ \\
$\tau_{\nu}$ & 9000 & $3.10^{6}$ & $2.10^{6}$ & $4.10^{6}$ & $\mathrm{~ns}$ \\
$\tau_{r}$ & 14 & 4 & 4 & 5 & $\mathrm{~ns}$ \\
$\tau_{e q}$ & 42 & 0.4 & 0.4 & 0.7 & $\mathrm{~ns}$
\end{tabular}

\section{Scaling Parameters}

\begin{tabular}{l|c|c|c|c|}
$\mathbb{M}$ & 14 & 4.1 & 1 & 18 \\
$\mathbb{M}_{A}$ & 1.2 & 0.9 & 0.4 & 1.7 \\
$\beta_{d y}$ & 2.8 & 1.8 & 0.3 & 5.9 \\
$\beta_{t h}$ & $8.10^{-3}$ & $6.10^{-2}$ & 0.2 & $1.10^{-2}$ \\
$\mathbb{P}$ & 0.6 & 59 & 9.5 & 290 \\
$\mathbb{R}_{m}$ & 75 & 1.6 & 1.1 & 1.9 \\
$\mathbb{R}$ & 9000 & $4.10^{5}$ & $9.10^{4}$ & $2.10^{6}$ \\
$\mathbb{K}$ & $5.10^{-3}$ & $4.10^{-4}$ & $7.10^{-4}$ & $4.10^{-4}$ \\
$\mathbb{H}_{i}$ & 0.2 & $1.10^{-3}$ & $1.10^{-3}$ & $2.10^{-3}$ \\
$\mathbb{H}_{e}$ & 140 & 0.8 & 0.9 & 1
\end{tabular}

Table 1: Measured and estimated plasma conditions with plasma expansion gradient length scale $L=1 \mathrm{~mm}$ and magnetic field $B=$ $20 \mathrm{~T}$ for 3 cases: 1) the initial plasma expansion, 2a) the expanding cavity region, 2b) the collapsed cavity and 3) the jet far from the cavity. The derivation of the plasma parameters is explained in the text.
To understand the plasma collisionality, we calculate the electron $\left(\tau_{e i}\right)$, ion $\left(\tau_{i i}\right)$, and electron-ion equilibration $\left(\tau_{e q}\right)$ inverse collision rates using values from Braginskii[56]. Here and in further calculations in this Article, we take the Coulomb $\operatorname{logarithm} \log \Lambda$ as 10 . The mean free paths $\lambda_{\alpha}=v_{T \alpha} \tau_{\alpha}$ are calculated using the thermal speeds $v_{T \alpha}$ and the inverse collision rates $\tau_{\alpha}$.

$$
\begin{array}{r}
\tau_{e i}=\frac{12 \pi^{3 / 2} \epsilon_{0}^{2} m_{e}^{1 / 2} T_{e}^{3 / 2}}{\sqrt{2} Z n_{e} q_{e}^{4} \ln \Lambda} ; \quad \tau_{i i}=\tau_{e i} \sqrt{\frac{2 m_{i}}{m_{e}}} Z^{-2} \\
\tau_{e q}=\tau_{i i} \sqrt{\frac{m_{i}}{m_{e}}}=\tau_{e i} \frac{\sqrt{2} m_{i}}{m_{e}} Z^{-2}
\end{array}
$$

From the short electron-ion equilibration times as shown in Table 1 we find that for most of the plasma conditions the plasma is collisional enough to justify an equilibration between ion and electron temperatures. The exception to this is that during the initial expansion of the plasma, there will be a hot $(80 \mathrm{eV})$, low density region $\left(10^{17} \mathrm{~cm}^{-3}\right)$, where this equilibration time may be long.

\subsection{Dimension-less Scaling Parameters}

To characterize the plasma in terms of the importance of advection with respect to thermal, magnetic and viscous diffusion we follow the treatments of Ryutov et al. in Refs. [13, 12].

The advective term $\chi_{a d}=L v$ is given by the gradient length scale $L$ and the characteristic velocity $v$. This characteristic velocity is taken as the greater of the bulk velocity $u_{d}$ and the sound speed $C_{s}$. In our calculations we define $L=1 \mathrm{~mm}$, as this is generally the distance over which gradients are observed in the plasma density (see Fig. 5).

Heat diffusion is characterized by Braginskii[56] as $\chi_{h e}=$ $\kappa_{e} / \rho C_{v}$, where $\kappa_{e}$ is the thermal conduction coefficient. We use the (unmagnetized) formulation from Ryutov et al. [13] :

$$
\chi_{h e}\left[\mathrm{~m}^{2} / \mathrm{s}\right] \simeq 2 \times 10^{17} \frac{T_{[\mathrm{eV}]}^{5 / 2}}{\ln \Lambda Z(Z+1) n_{i\left[\mathrm{~cm}^{-3}\right]}}
$$

The magnetic diffusion is defined as $\chi_{m}=\eta \mu_{0}^{-1}$, where the electrical resistivity is $\eta=m_{e} / n_{e} q_{e}^{2} \tau_{e i}$. The viscosity is given by $\chi_{\nu}$. These are characterized in Ryutov et al. [12, 13] as:

$$
\begin{array}{r}
\chi_{m}\left[\mathrm{~m}^{2} / \mathrm{s}\right] \simeq 1.5 \times 10^{3} Z T_{[\mathrm{eV}]}^{-3 / 2} \\
\chi_{\nu}\left[\mathrm{m}^{2} / \mathrm{s}\right] \simeq 2 \times 10^{15} \frac{T_{[\mathrm{eV}]}^{5 / 2}}{\ln \Lambda \sqrt{\frac{m_{i}}{m_{p}}} Z^{4} n_{i\left[\mathrm{~cm}^{-3}\right]}}
\end{array}
$$

To quantify these numbers in the context of the experiment, we use the gradient length scale $L$ to determine a relative timescale $\tau=L^{2} / \chi\left(\right.$ e.g. $\left.\tau_{a d}=L^{2} / \chi_{a d}\right)$ above which these effect will be important. 
In order to define the relative importance of the diffusive terms to advection and to understand how this experiment would scale to astrophysical phenomena, we calculate the non-dimensional Peclet $\left(\mathbb{P}=\chi_{a d} / \chi_{h e}\right)$, magnetic Reynolds $\left(\mathbb{R}_{m}=\chi_{a d} / \chi_{m}\right)$ and Reynolds $\left(\mathbb{R}=\chi_{a d} / \chi_{\nu}\right)$ numbers. These terms reveal the relative importance of advection versus heat diffusion, magnetic diffusion and viscosity, respectively. When these numbers are large, advection dominates.

To understand the importance of the motion of individual particles, as opposed to bulk fluid motion, we define the localization parameter $\mathbb{K}=l^{*} / L$ from Ref. [13], which is defined as the ratio of the local length scale $l_{*}$ to the characteristic gradient length scale $L$. The local length scale is defined as the lesser of the mean free path and the gyroradius for each species. For our calculations we use the largest length scale between the ion and electron species. This can be thought of as a generalized Knudsen number; thus when $\mathbb{K} \ll 1$ kinetic and finite gyroradius effects can be neglected. In all of the cases considered here we find a small localization parameter, which means that the plasma is sufficiently collisional to be treated as a fluid.

To understand the role of magnetization versus collisional effects, we calculate the Hall parameter $\mathbb{H}_{\alpha}=\omega_{c \alpha} \tau_{\alpha}$ for the species $\alpha$. This number is representative of the number of cyclotron orbits a particle performs before undergoing a collision. Thus for high Hall numbers, we consider the plasma to be strongly magnetized. We find that for these plasma conditions the ions are not substantially magnetized $\left(\mathbb{H}_{i} \ll 1\right)$. On the other hand, the electrons are considerably magnetized $\left(\mathbb{H}_{e} \gg 1\right)$ at high temperatures and have marginal magnetization $\left(\mathbb{H}_{e} \sim 1\right)$ at lower temperature (i.e. in the far jet region).

Finally, we define the plasma beta. This value relates the relative importance of the total kinetic pressure of the plasma to the magnetic pressure $P_{m}=B^{2} /\left(2 \mu_{0}\right)$. We define the plasma beta in two ways, first the dynamic beta $\beta_{d y}=P_{d y} / P_{m}$, which is the ratio of the ram pressure $P_{d y}=\rho_{i} u_{d}^{2}$ to the magnetic pressure. Secondly, the thermal beta $\beta_{t h}=P_{t h} / P_{m}$, which is the ratio of the thermal pressure $P_{t h} \simeq n_{e} T_{e}$ to the magnetic pressure. Throughout the evolution of the jet, the balance between magnetic and kinetic pressure is dynamically changing. In the cavity region, the plasma is strongly magnetized, while the jet far from the target is dominated by the plasma pressure and is unmagnetized.

\subsection{Radiative Cooling}

The radiative cooling power was found using the code FLYCHK [55] for different ion densities $n_{i}$ in the range from $10^{17}$ to $10^{18} \mathrm{~cm}^{-3}$. For each density the total (boundbound, bound-free and free-free) radiation power was found for both Carbon $\left(P_{r C}\right)$ and Fluorine $\left(P_{r F}\right)$ considering that their densities were $\frac{1}{3} n_{i}$ and $\frac{2}{3} n_{i}$, respectively. The total radiation power density was then calculated as a summation of these two powers, $P_{r}=P_{r C}+P_{r F}$ at electron temperature range $T_{e}=20-100 \mathrm{eV}$ giving the values of $\sim 5 \times 10^{-9} \mathrm{~J} / \mathrm{s} /$ atom. We realize that a true multi-species treatment of the radiation would be the proper way to calculate this, however for an order-of-magnitude estimate we expect that this is sufficient. The radiative cooling rate is on the order of $10 \mathrm{eV} / \mathrm{ns}$ and the radiative cooling time is on the order of few ns as estimated in the same $T_{e}$ range.

The cooling rate of the plasma $\left(\Delta T_{e} / \Delta t=P_{r} / C_{v}\right)$ was found using the radiative cooling power and the heat capacity $C_{v}$ of the plasma, as determined from Livermore equation-of-state (LEOS) tables. The radiative cooling time $\tau_{r}=0.1 \times T_{e} /\left(\Delta T_{e} / \Delta t\right)$ was evaluated as the amount of time for the temperature to decrease by $10 \%$ at the given cooling rate. We note here that the optical skin depth of the plasma is quite long and thus we expect the plasma to be optically thin.

However, it is necessary to note that the estimations of the radiative cooling power were made considering a plasma in the local thermodynamic equilibrium (LTE). For low-temperature (few tens of eV) recombining plasma the radiative bound-bound transitions should provide sufficient increase in radiative cooling rate and corresponding shortening of the radiative cooling time. So the values given above represent a definite underestimation. In fact, as shown in Table 1, one may consider the radiative cooling to play an important role in the present experiments, because the plasma is recombining all along the range of the jet propagation away from the target surface.

\subsection{Plasma Parameters Summary}

At early times (Region 1), Table 1 suggests that the plasma will be highly magnetized $\left(\mathbb{H}_{e} \gg 1\right)$ and that the magnetic field is effectively frozen-in with the plasma flow $\left(\mathbb{R}_{m} \gg 1\right)$. The $\beta_{d y}$ is above unity, suggesting that at this point the radial component of the plasma pressure is able to push against the magnetic field and will strongly modify the topology of the field.

At later times, still close to the target (Region 2) $\beta_{d y}$ drops below unity, indicating an increased ability of the magnetic field to push back against the plasma flow. The lowered $\mathbb{P}$ suggests that heat diffusion in this region can be neglected due to the cooling down of the plasma. While still greater than unity, the decreasing value of $\mathbb{R}_{m}$ suggests that magnetic diffusion may play some role in the dynamics.

Finally, at later times and distances far from the target, we find that the Mach-number is high $\left(\mathbb{M} \sim 18, \mathbb{M}_{A} \sim 1.7\right)$ and that the strong ram pressure maintains a high $\beta_{d y}$. However, the role of the magnetic field here will be relatively small. The electrons are only marginally magnetized $\left(\mathbb{H}_{e} \sim 1\right)$. While $\mathbb{R}_{m}$ is not particularly small, the magnetic diffusion time $\left(\tau_{m} \sim 5 \mathrm{~ns}\right)$ is low compared to the time of interest $(60 \mathrm{~ns})$. This indicates there has been plenty of time for the magnetic field to diffuse into the plasma.

Other than at very early times $(<16 \mathrm{~ns})$ the ionelectron equilibration time $\left(\tau_{e q}\right)$ is less than $1 \mathrm{~ns}$, which 


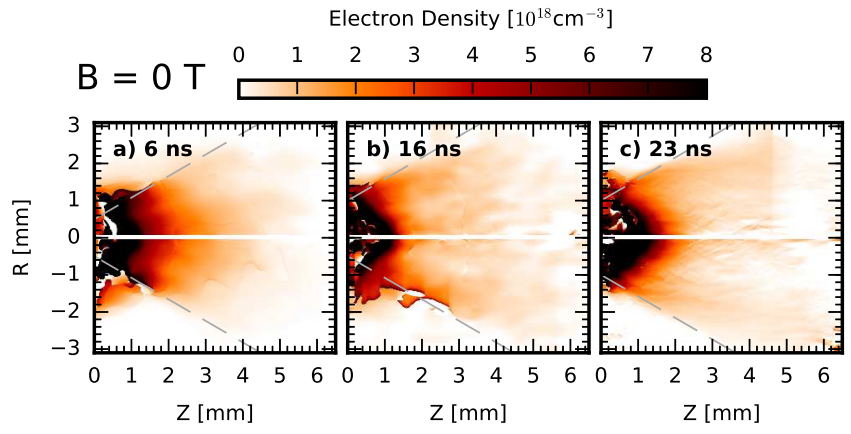

Figure 4: Pseudo-color images of electron density taken via interferometry showing the electron density from a $2 \times 10^{13} \mathrm{~W} / \mathrm{cm}^{2}$ laser irradiating a $\mathrm{CF}_{2}$ target without an ambient magnetic field. The central \pm 5 pixels $( \pm 55 \mu \mathrm{m})$ have been removed due to the uncertainty of the Abel inversion on axis. The dashed lines show a $30^{\circ}$ half-angle.

suggests that our assumption of equal ion and electron temperatures is valid. Also, at all times, we find that the radiation time $\left(\tau_{r}=4-14 \mathrm{~ns}\right)$ is relatively short compared to the evolution of the plasma, which suggests that the plasma is cooling substantially as time progresses. This cooling of the plasma is important as it reduces the sound speed of the plasma and thus increases the Mach-number.

\section{Characterization of a Large Aspect Ratio Jet}

\subsection{Electron Density Evolution without Magnetic Field}

To characterize the ablation of plasma without an applied B-field, the $2 \times 10^{13} \mathrm{~W} / \mathrm{cm}^{2}\left(E_{L}=40 \mathrm{~J}\right)$ laser irradiated a $\mathrm{CF}_{2}$ target without powering the Helmholtz coil $(B=0 \mathrm{~T})$. Fig. 4 shows the electron density, as diagnosed via interferometry, for three different timings as expanding away from the initial target surface at $z=0$. In all of the interferometry images, the electron density is found via Abel inversion, which is done separately for the top and the bottom images. As numerical errors from the Abel technique lead to a high uncertainty on-axis the central region \pm 5 pixels have been excluded from the image. Noise in the fringes leading to difficulty in the phase inversion is responsible for many of the sharp and small scale features in the images, especially at the transition between the plasma and the vacuum. One can compare the upper and lower portion of each image to get a better understanding of the features caused by this noise.

Notice that in Fig. 4 none of the images show any collimation of the plasma and it expands in a wide-angle flow with a half-angle of around $30^{\circ}$. This wide flow is expected and has been observed much earlier [57]. The expansion of such a non-magnetized flow is generally characterized as a planar (i.e. 1D) expansion close to the target $\left(z \sim R_{\text {spot }}\right)$ and as a quasi-hemispherical (i.e. 3D) expansion at distances far from the target $\left(z \gg R_{\text {spot }}\right)$ [58, 59], as we observe experimentally. We note that some previous work has referred to these flows as "jets" $[60,61]$ due to their modest directionality. However, these flows are do not have high-aspect ratios and thus the authors themselves have deemed such a term inappropriate[62]. As cited in the introduction, unmagnetized jet formation is possible using shaped targets[40, 43] or multi-material layers[41, 42, 44] to hydrodynamical shape the flow into a jet. However, none of these techniques were used in the present setup and thus we observe a widely diverging flow without the formation of a jet.

\subsection{Electron Density Evolution with 20 T Magnetic Field}

The dynamics of the plasma expansion change considerably with the addition of the external $20 \mathrm{~T}$ magnetic field. Figure 5 shows the electron density evolution, measured via interferometry, over many millimeters and many tens of nanoseconds in the case of the irradiation of a $\mathrm{CF}_{2}$ target with a $2 \times 10^{13} \mathrm{~W} / \mathrm{cm}^{2}$ laser in an $20 \mathrm{~T}$ applied magnetic field. These images were taken over multiple shots with nominally identical setups. To capture different frames in time, the delay between the interferometer probe beam and the main laser pulse was varied from 6 to 70 ns. To capture long spatial scales in the plasma, the target was recessed within the coil; different images are shown separated by dotted lines in Fig. 5. Comparing the cases with (Fig. 5) and without (Fig. 4) magnetic fields, it is very clear that the $20 \mathrm{~T}$ field drastically changes the plasma evolution, and that the field acts to confine and collimate the plasma into a narrow jet.

If we consider the front edge of the jet, we find a jet velocity $u_{d} \sim 1000 \mathrm{~km} / \mathrm{s}$ at early times by noticing that the plasma has progressed to $6 \mathrm{~mm}$ over a time of $6 \mathrm{~ns}$. This is consistent with the non-magnetized case and shows that the velocity of at the head of the jet is not particularly faster in the presence of the magnetic field. However, due to strong collimation, the jet has a much higher density at distances further from the initial target surface. Considering the sound speed $(70 \mathrm{~km} / \mathrm{s})$ calculated previously, we find that the jet is propagating at high Mach number $(\mathbb{M}=14)$ at this leading edge of the jet.

We now describe the evolution of the jet (as sketched in Fig. 1b) based on direct experimental observations and from our understanding of the physics supported by simulations (similar to those in Refs.[1, 2]). During the evolution of the jet there are three distinct spatial regions, which evolve in different ways and result from an interplay between kinetic plasma pressure and magnetic pressure. These are the cavity, the conical shock and the jet. We label these regions in Fig. 5b. Here the cavity region extends from around 1 to $4 \mathrm{~mm}$ from the initial target surface, the conical shock region is from 4 to $6 \mathrm{~mm}$, and the collimated jet is from $6 \mathrm{~mm}$ and greater.

The cavity region is given its name due to the lower density on the inside compared to the high density region surrounding it, as is shown clearly in the early time frames of Fig. 6. We see also that the density is higher on axis as compared to the case without B-field. The sharp radial density gradient in that case is formed as the ram pressure 

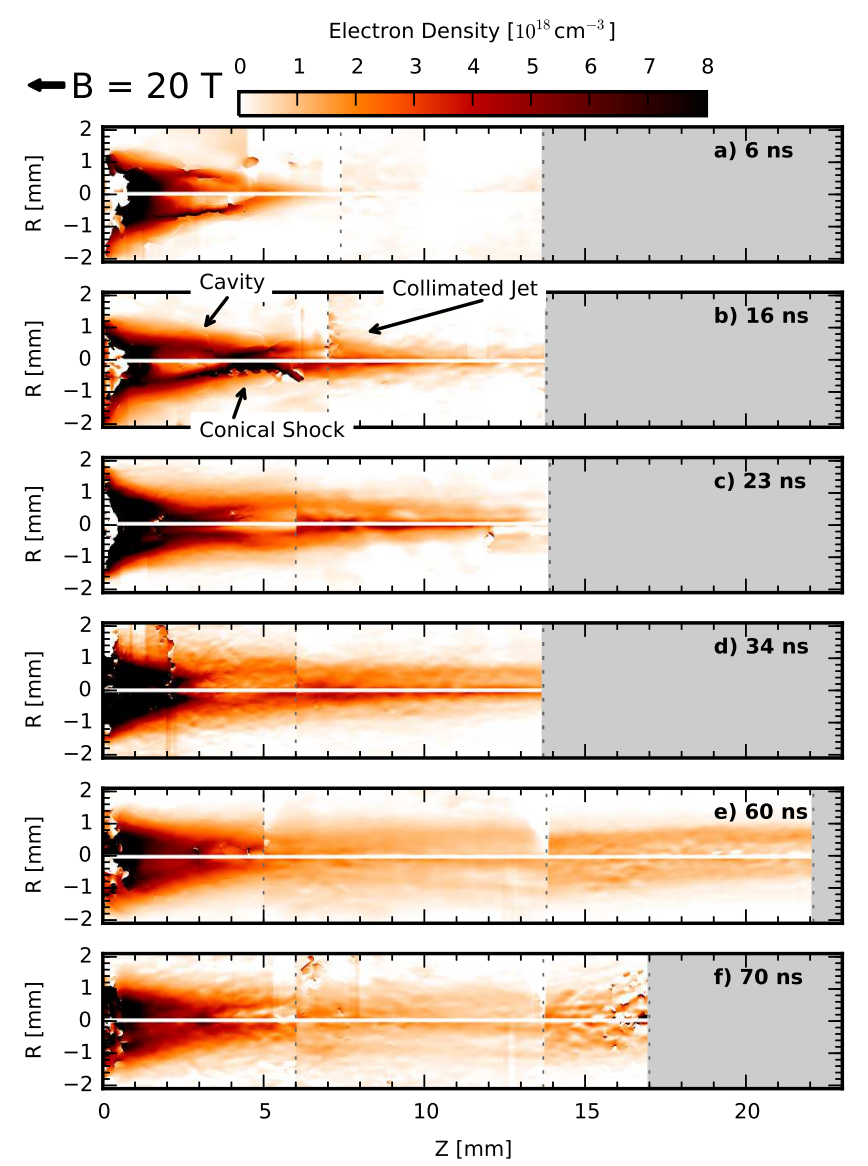

Figure 5: Pseudo-color images of electron density taken via interferometry showing jet propagation from 6 to $70 \mathrm{~ns}$ as indicated in the plots. The jet was created by a $2 \times 10^{13} \mathrm{~W} / \mathrm{cm}^{2}$ laser irradiating a $\mathrm{CF}_{2}$ target in a $20 \mathrm{~T}$ ambient magnetic field. The dotted vertical lines indicate the edges of the individual images. The gray background indicates a region where no data was taken or the fringes were not of sufficient quality to be unwrapped properly. The central \pm 5 pixels $( \pm 55 \mu \mathrm{m})$ have been removed due to the uncertainty of the Abel inversion on axis. Note that many of the fine structured features are due to noise in the fringes of the interferometer and thus are non-physical. These areas are most notable around the target, in (a) at $z=3-5 \mathrm{~mm}, r=-0.5 \mathrm{~mm}$, in (d) at $z=2 \mathrm{~mm}, r=0-2 \mathrm{~mm}$ and in (f) at $z>15 \mathrm{~mm}$ ). The upper and lower frames in the images represent Abel inversion taken from either side and comparison between the upper and lower portions can be used to ascertain the experimental uncertainty.

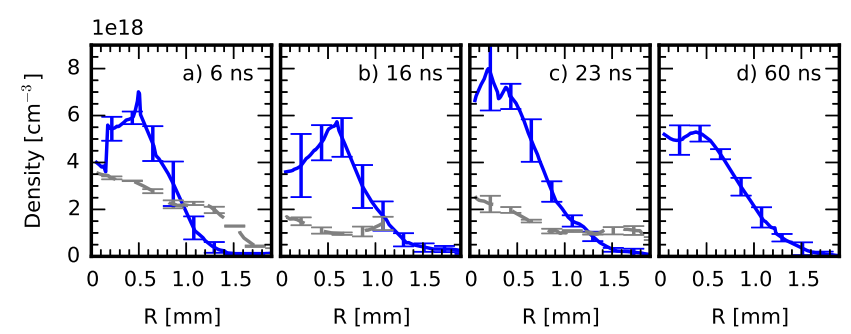

Figure 6: The radial electron density profile of the jet at a distance of $2.0 \mathrm{~mm}$ from the initial target surface (averaged over $\pm 55 \mu \mathrm{m}$ ). Solid lines show the case with a $20 \mathrm{~T}$ applied magnetic field and dotted lines show cases with no applied field. The time after laser irradiation is shown in each plot. Error bars are shown at even intervals.
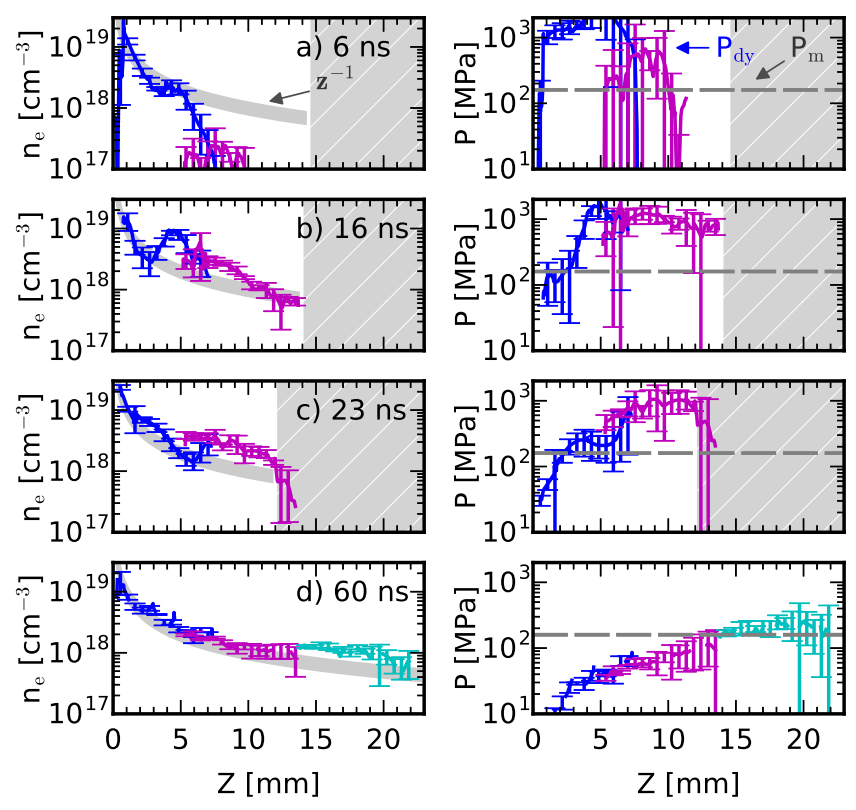

Figure 7: (Left) Longitudinal electron density, $n_{e}$, near the center $(110 \mu \mathrm{m}<r<270 \mu \mathrm{m})$ of the jet at times 6-60 ns after laser irradiation. The lines show the cases with an external $20 \mathrm{~T}$ field. The different shades of the solid lines are for different shots taken with different recession distances of the main target to look at different regions of the jet. The solid gray fill-in is $n_{e} \propto z^{-1}$ with $\pm 20 \%$ and provides a reference to the fall off of the data. The solid gray hashed fill shows regions where no data was taken or the data is of poor quality. Error bars in the data are shown at even intervals. (Right) Ram pressure, $P_{d y}$, (solid lines) and nominal magnetic pressure, $P_{m}$, (dashed line) at the same times.

of the plasma pushes out against the magnetic field lines to create a shock[1]. This will transfer transverse ram pressure into thermal pressure (i.e. heating) of the ions. Figure 6 shows the evolution of the radial profile of the cavity at a distance of $2 \mathrm{~mm}$ from the target surface. At early times there is a strong increase in density at the edges that is representative of the shock from the plasma pushing against the magnetic pressure.

This oblique shock at the edge of the cavity is then responsible for re-directing the flow of plasma along the shock so that it follows the contour of the cavity until it converges on the axis (see Fig. 1b). This convergence of the flow on axis generates a conical shock[63] at the longitudinal tip of the cavity $(z \sim 4 \mathrm{~mm}$ at $16 \mathrm{~ns})$, which then re-directs the flow again. Since the plane of the conical shock is along the $z$-axis, the flow is symmetrically refracted along the $z$-axis and a collimated jet is formed. This cavity can be thought of as a nozzle, a poloidal magnetic nozzle, where the oblique shock and conical shocks create the walls of the nozzle. However, one major difference between this type of collimation and a mechanical nozzle is that the main acceleration mechanism is the initial energy from the absorbed laser and not the constriction of the flow through the throat of the nozzle, a distinction due to the fact that the experiment is not in steady-state. 
At later times we see the narrow, focused jet propagate over long distances $(>23 \mathrm{~mm},>10: 1$ aspect ratio) and times ( $>70 \mathrm{~ns}, 100 \mathrm{x}$ the laser duration). From the plasma jet parameters at $z=22 \mathrm{~mm}$ and $t=60 \mathrm{~ns}$, we infer a sound speed of $C_{s}=20 \mathrm{~km} / \mathrm{s}$ and thus a Mach number $\mathbb{M}=18$ in Table 1 . At this location the plasma is only marginally conductive $\left(\mathbb{R}_{m} \sim 1\right)$ and the magnetic diffusion time is around 5 ns. This suggests that strong magnetic field gradients cannot exist and that the magnetic field cannot be responsible for collimating the jet in this region. Additionally, the high $\beta_{d y}$ at this point indicates that the magnetic pressure would not be able to hold in the flow it were not already collimated. Thus the jet must be propagating hydrodynamically and we expect a divergence angle $\theta=\tan ^{-1}(1 / \mathbb{M}) \simeq 3^{\circ}$ (18:1), which is consistent with observations. Additionally, considering that a peak bulk velocity of $1000 \mathrm{~km} / \mathrm{s}$ was observed at early times, it is reasonable to expect a peak Mach-number of 50 at the leading edge of the jet, which would lead to $\theta \simeq 1^{\circ}$ (50:1). Future studies looking at distances much further along the jet would be useful to investigate the full aspect ratio at the tip of the jet.

We now look quantitatively at the longitudinal profile of the electron density near the center of the jet in the left side of Fig. 7. Here the different shaded lines represent different shots taken as the target was recessed into the coil to capture a large spatial region. The overlap between the lines highlights the repeatability of the formation of the jet over different shots at the same nominal laser parameters. With an applied field, the profile shows a strong bump in the density a few millimeters from the base of the jet. This region is the conical shock that is caused by material that is re-directed from the edges of the cavity back into the center as explained earlier. At distances far from this shock we see an expansion that follows a scaling that is consistent with a 1D Lagrangian ballistic expansion with a constant ionization fraction. Due to the flatter falloff of the spatial dependence at distances far from the target surface we see that at $60 \mathrm{~ns}$ (Fig. $7 \mathrm{~d}$ ) the jet maintains a relatively constant density $\left(n_{e} \simeq 10^{18} \mathrm{~cm}^{-3}\right)$ for over a long distance $(5-20 \mathrm{~mm})$ and could potentially be used as a constant density medium for experiments probing plasma parameters at these conditions. The right side of Fig. 7 shows the ram and nominal magnetic (i.e. $20 \mathrm{~T}$ ) pressures. The ram pressure, $P_{d y}=\rho_{i} u_{d}^{2}$, is found assuming that the plasma expansion is ballistic (i.e. $u_{d}=z / t$ ) and that the plasma has a constant average charge state of $\bar{Z}=6$, as consistent with the plasma parameters estimated earlier in Table 1. From these plots it seems clear that the ram pressure is the dominant force at the tip of the jet at all times.

To understand the interplay between the forces exerted by the plasma and those exerted by the magnetic field, we plot the longitudinal location of the tip of the cavity as a function of time in Fig. 8. These values are calculated from the location of the peak density of the conical shock region and taking the width of this region as the uncertainty.

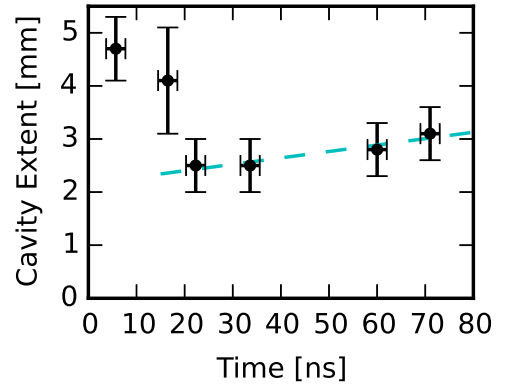

Figure 8: The location, in the $z$-direction, of the tip of the cavity (i.e. conical shock region) at different times. The values are determined from the location of the peak density of the shock and the uncertainty is defined as the width of the shock. A linear fit of the expansion at later times is shown as the dotted line, which has a slope corresponding to $12 \mathrm{~km} / \mathrm{s}$.

Considering that the plasma must initially start at the target surface (i.e. $z=0 \mathrm{~mm}$ at $t=0 \mathrm{~ns}$ ), this plot shows that the cavity expands at early times ( $\leq 16 \mathrm{~ns})$, then quickly recesses $(\sim 23 \mathrm{~ns})$ and finally expands again at a slower pace $(\geq 23 \mathrm{~ns})$. The initial expansion and recession is consistent with the evolution of the ram pressure $P_{d y}$ compared with the nominal magnetic pressure $P_{m}$. At early times ( $<10 \mathrm{~ns}), P_{d y}$ is large as it corresponds to the fastest leading edge of the plasma expansion. Thus at this time $P_{d y}$ exceeds $P_{m}$ and the plasma pushes out against the magnetic field to reach a peak of around $4.5 \mathrm{~mm}$ at 5-15 ns. As time goes on ( $>10 \mathrm{~ns})$, the velocity of the outflow near the cavity decreases and $P_{d y}$ falls below $P_{m}$ causing the cavity to be pushed back by the magnetic field to a position of around $2 \mathrm{~mm}$ at $20 \mathrm{~ns}$.

We note once again that while the initial force from the B-field will be in the radial direction (i.e. due to the longitudinal field lines), the field line topology will be significantly modified as the plasma evolves. This will create a radial component to the magnetic field and will result in a significant force in the longitudinal direction. Our simulations[1] suggest that this radial force will result in an effective pressure of $10-50 \%$ of the nominal (160 MPa) value. The final (> $20 \mathrm{~ns}$ ) expansion of the cavity is quite slow and a best fit to the data corresponds to an expansion velocity of $12 \pm 10 \mathrm{~km} / \mathrm{s}$. The dynamics behind this late time evolution should be driven by both the plasma ram and thermal pressure considering that $u_{d}$ and $C_{s}$ are of the same order (i.e. from Table 1, Region $2 \mathrm{~b}$ ). This dynamic is also complicated by the fact that $\mathbb{R}_{m}$ is near unity suggesting that magnetic diffusion may be of some importance during this period (i.e. from 20 to $60 \mathrm{~ns}$ ).

\subsection{Optical Emission from the Jet}

The optical emission originating from the central region $(r<100 \mu \mathrm{m})$ of the jet is shown in the case without (Fig. 9a) and with (Fig. 9b) an applied 20 T magnetic field. The emission is streaked so that the downward direction is the time from the main pulse irradiation and the horizontal axis is the longitudinal direction away from the target 


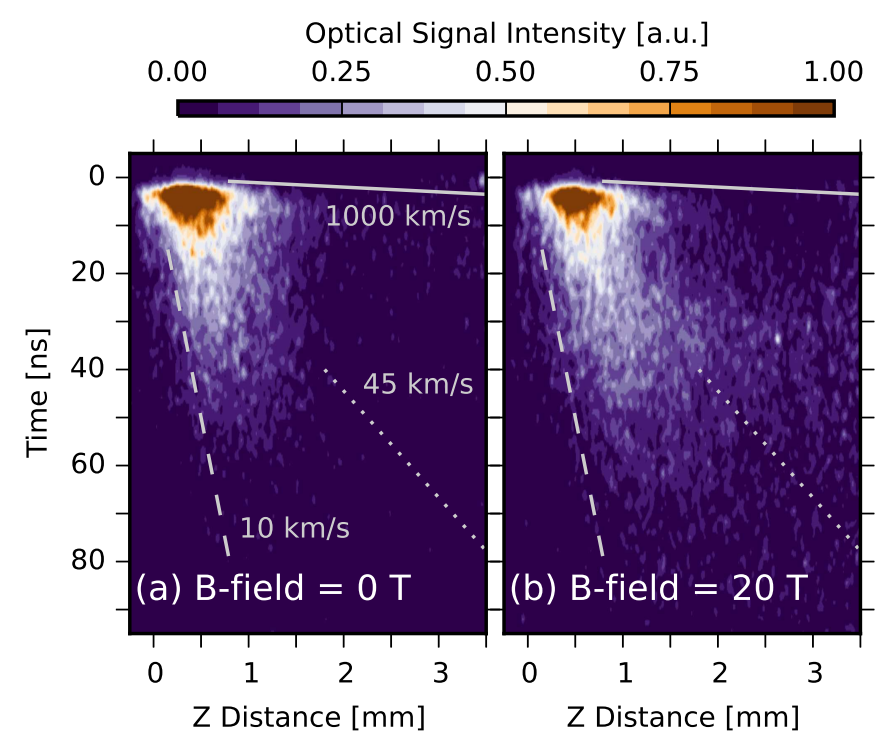

Figure 9: Optical emission from the streaked optical imager (SOI) showing the cases (a) without and (b) with a $20 \mathrm{~T}$ applied magnetic field. The vertical axis is the time from the main pulse irradiation and the horizontal axis is the longitudinal distance from the target surface. The signal intensity units are arbitrary but are the same on both images. The peak intensities are (a) 2.6 and (b) 1.6 for the cases with and the without applied field, respectively. The overlaid dashed, dotted and solid lines are the same on both pots and have velocities of 10,45 and $1000 \mathrm{~km} / \mathrm{s}$, respectively, and pass through the origin.

in space. A dashed line is plotted on top of the images that shows a velocity of $10 \mathrm{~km} / \mathrm{s}$, which seems to roughly correspond with the darkening of the image at distances near to the target surface. The reason for this darkening is likely due to the expansion of high density plasma that is optically thick and thus blocks emission coming from the center of the plasma.

The emission in the case without a magnetic field decreases monotonically both in time and in space. By around $50 \mathrm{~ns}$ the emission has fallen below the noise level of the diagnostic, and in the longitudinal direction the emission has decreased to below the noise level at a distance of $2 \mathrm{~mm}$.

On the other hand, with an applied magnetic field, the emission extends to both longer times $(\sim 80 \mathrm{~ns})$ and over larger distances $(\sim 3 \mathrm{~mm})$. Also, in contrast to the case without field, the emission does not decrease monotonically. In fact, the emission at distances greater than $1.5 \mathrm{~mm}$ starts to increase at around $25 \mathrm{~ns}$. This can be attributed to heating from the conical shock as it relaxes backwards towards the target. This is consistent with both the timing and the position of the tip of the cavity shown in Fig. 8.

\subsection{Electron Temperature via FSSR X-ray Spectrometry}

The time-integrated electron temperatures and densities from the FSSR are shown in Fig. 10 for the cases with and without an applied $20 \mathrm{~T}$ B-field. First of all, we
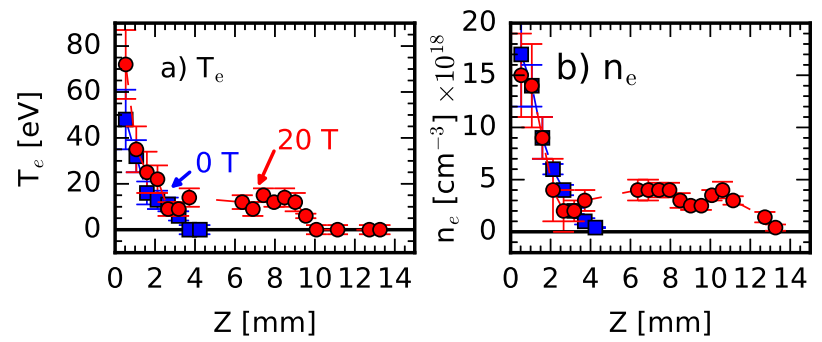

Figure 10: Experimental data from the FSSR showing emissionweighted a) electron temperature and b) electron density for the cases without (squares) and with (circles) a $20 \mathrm{~T}$ magnetic field.

note that Fig. 10b gives electron densities that are consistent with those observed via interferometry, thus giving us confidence in our diagnostics and analysis techniques. As in the interferometry data, the electron density decreases monotonically in the case without B-field, but shows an extended density profile up to $10 \mathrm{~mm}$ in the case with an applied $20 \mathrm{~T}$ field.

The electron temperature in Fig. 10a peaks at 50-80 eV and then decreases with distance from the target. In the case without B-field, the temperature drops below the diagnostic resolution around $3 \mathrm{~mm}$. On the other hand, with a 20 T B-field the electron temperature drops to $\sim 10 \mathrm{eV}$ around $4 \mathrm{~mm}$ and then stays relatively constant for many millimeters. Another feature in the $20 \mathrm{~T}$ case is a small increase in temperature around $1.5-2 \mathrm{~mm}$ as compared to the $0 \mathrm{~T}$ case. This location is similar to the location of the cavity after its early collapse around 20 ns as seen from interferometry (see Fig. 8) and with the increased optical emission from the SOI; this is consistent with the idea that the conical shock at the tip of the cavity leads to increased heating[2].

\section{Fidelity of Jet Creation Across a Variety of Plasma Conditions}

To explore the fidelity of plasma collimation across a variety of plasma conditions, we varied the laser energy incident on the target, the target material and the direction of the magnetic field with respect to the target. Each of these variations show quantitative differences in jet and cavity formation, however the qualitative behavior is similar across the variety of conditions, which highlights the repeatability of the phenomena.

\subsection{Variable Laser Intensity}

The laser intensity was adjusted by varying the laser energy from 3, 6 and $16 \mathrm{~J}$ corresponding to laser intensities of $1.3,2.6$ and $6.9 \times 10^{12} \mathrm{~W} / \mathrm{cm}^{2}$, respectively. The electron densities of these plasmas due to the different irradiation conditions are shown in Figure $11 \mathrm{a}$ at 10 and 28 ns. At early times (10 ns) the differences between the various cases are clear as both the radial and lateral extents of the cavity increase as a function of the laser intensity. At the later time (28 ns), all of the plasmas have 
Electron Density $\left[10^{18} \mathrm{~cm}^{-3}\right]$

$\begin{array}{lllllllll}0 & 1 & 2 & 3 & 4 & 5 & 6 & 7 & 8\end{array}$

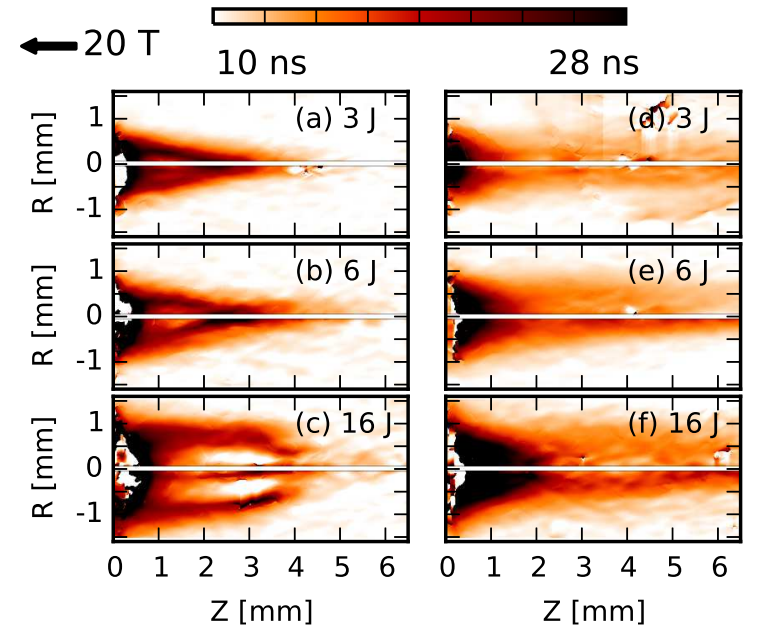

Figure 11: (a-f) Electron density for laser irradiation energies of (top) $3 \mathrm{~J}$, (middle) $6 \mathrm{~J}$ and (bottom) $16 \mathrm{~J}$. The left side corresponds to $10 \mathrm{~ns}$ and the right side to $28 \mathrm{~ns}$ after the main pulse irradiation.

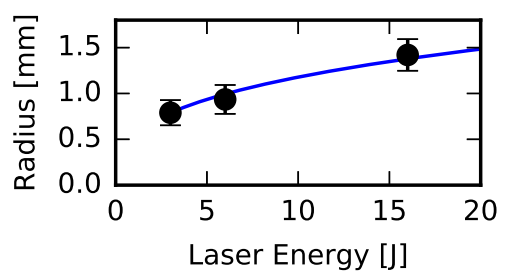

Figure 12: Circles show the cavity radius (at 10\% of the peak density) at $10 \mathrm{~ns}$ at $z=1 \mathrm{~mm}$ from the target for the various laser irradiation energies. Error bars are defined as the difference between the radius at $50 \%$ and $10 \%$ of peak density. The solid line is from eq. (7) with $f=12 \%$.

formed a high-density region close to the target with a collimated jet extending over a long distance. Thus while there are quantitative differences among the jets, the qualitative form remains the same.

To understand how increasing the kinetic energy of the plasma changes its expansion in the magnetic field, the radial extents of the cavity for the different cases are plotted as a function of laser energy in Fig. 11b. The values are taken at the location where the density reaches $10 \%$ of the peak density at a distance of $z=1 \mathrm{~mm}$ from the target at $10 \mathrm{~ns}$.

We expect that the maximum radial extent of the cavity $R_{c}$ will vary as a function of the total plasma pressure $P_{k}=f \mathcal{E}_{L} / V$, where $f$ is the laser absorption, $\mathcal{E}_{L}$ is the laser energy and $V=\frac{2}{3} \pi R_{c}^{3}$ is the volume of a hemisphere, versus the magnetic pressure $P_{m}$. Here the laser absorption $f$ is simply the ratio of radially directed kinetic energy to laser energy and does not include other energy channels (e.g. radiation) and thus should not be used to understand the absolute laser absorption. Considering a hemispherical expansion this gives a relationship of $B^{2} / 2 \mu_{0}=f \mathcal{E}_{L} / \frac{2}{3} \pi R_{c}^{3}$

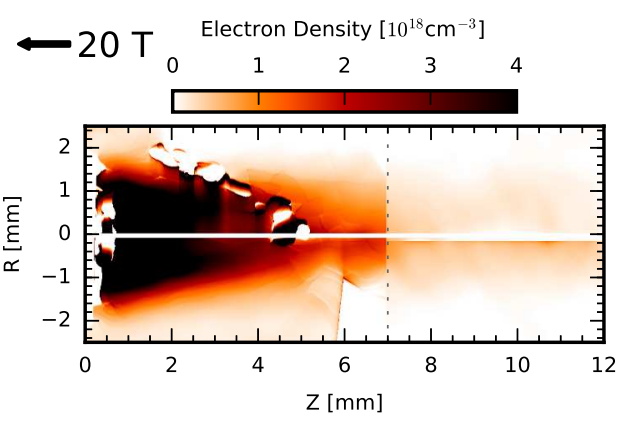

Figure 13: Electron density for the irradiation of a $\mathrm{Cu}$ target by the $2 \times 10^{13} \mathrm{~W} / \mathrm{cm}^{2}$ laser in a $20 \mathrm{~T}$ field at a time of $60 \mathrm{~ns}$.

and the solution for the radial extent $R_{c}$ :

$$
R_{c}=\left(\frac{f \mathcal{E}_{L}}{\frac{2}{3} \pi} \frac{2 \mu_{0}}{B^{2}}\right)^{1 / 3}
$$

The solid line in Fig. 12 represents eqn. (7) with a constant absorption fraction of $12 \%$ in agreement with our previous expectations[1].

\subsection{Target Composition}

We varied the composition of the target to investigate the feasibility of collimation using a material with a higher atomic number. As higher atomic number materials tend to emit more radiation they have been shown to form cooler and less divergent plasma expansions[42] (though not as high aspect ratio as the jets presented here). Figure 13 shows the electron density from the irradiation of a $\mathrm{Cu}$ target at $60 \mathrm{~ns}$ from the main pulse irradiation of $2 \times 10^{13} \mathrm{~W} / \mathrm{cm}^{2}$ in a 20 T field.

One might expect that the cooling of the plasma would decrease the amount of pressure in the plasma and thus decrease the size of the cavity at late times. However, this is not what we find experimentally. Instead at $1 \mathrm{~mm}$ away from the target the radius (at $10 \%$ of peak) for the $\mathrm{Cu}$ case is $1.7 \mathrm{~mm}$, while for the $\mathrm{CF}_{2}$ case (Fig. 5e) it is only $1.4 \mathrm{~mm}$ at the same timing. This difference could be due to a different number of factors. The most simple explanation is that $\mathrm{Cu}$ more efficiently absorbs the laser light and thus has more kinetic energy despite the fact that it may be cooling more strongly. Another explanation could be that the $\mathrm{Cu}$ plasma is cooler than the $\mathrm{CF}_{2}$ and that this leads a faster rate of magnetic diffusion, which thus creates less pressure pushing radially inwards against the plasma allowing it to expand further outward. Nonetheless, we observe that higher atomic number targets in a $20 \mathrm{~T}$ B-field still lead to collimation and jet formation that is sustained up to $60 \mathrm{~ns}$.

\subsection{Magnetic Field Alignment}

We investigated the effectiveness of axial magnetic collimation with a magnetic field that was not aligned perpendicular to the target. This was achieved by rotating 

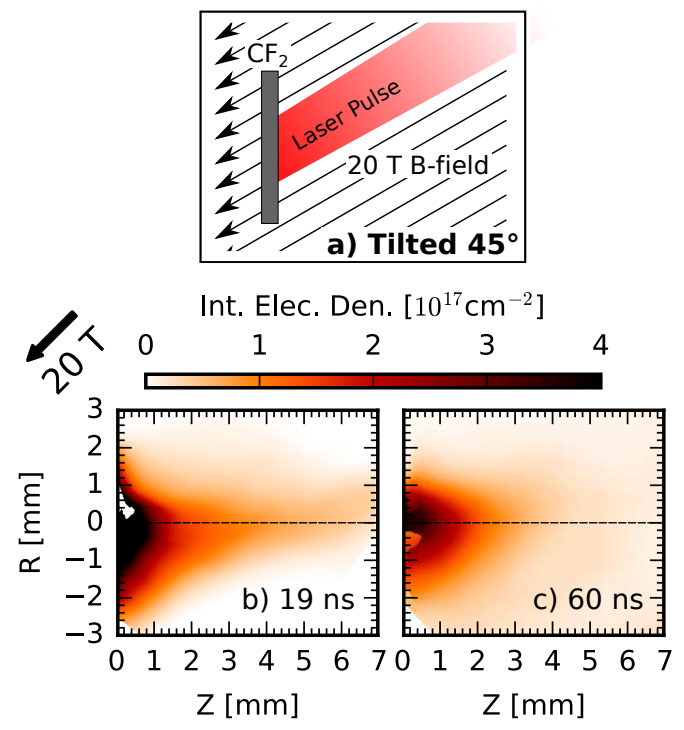

Figure 14: a) Schematic of the target setup from the point of view of the interferometer in with a magnetic field tilted by $45^{\circ}$. This angle was achieved by tilting the target in the experiment and then rotating the image after the shot. b,c) Pseudo-color images of lineintegrated electron density at $19 \mathrm{~ns}$ (b) and $60 \mathrm{~ns}$ (c) from laser irradiation with a $20 \mathrm{~T}, 45^{\circ} \mathrm{B}$-field. The dashed line in the images shows the middle of the laser spot.

the target by an angle of $45^{\circ}$ with respect to the magnetic field as shown in Fig. 14a. In this diagram, and in the following interferometry images, we have tilted the image by $45^{\circ}$ so that the target is in the same position as in the previous images.

We look at the evolution of the electron density in this configuration in Fig. 14b,c. We note that the Abel inversion method is not technically correct in this instance due to the lack of cylindrical symmetry. For this reason, we present the line-integrated electron density. At 19 ns (Fig. 14b) the plasma is redirected towards the axis, which is somewhat similar to the case with co-aligned magnetic field (Fig. 5b). However, in the $45^{\circ}$ case the plasma is compressed much closer to the target. Also, while we see a high-density region close to the target, it does not look as much like a "cavity" in that there is no low density region on axis and no high-density region at the edges. A difference that suggests that the formation of a strong oblique shock at the cavity edges is not as profound as in the cases where the field is un-tilted. Additionally, the asymmetry in the magnetic field has produced an up-down asymmetry in the electron density profile, where the plasma in the top half of the image is more confined on axis than in the bottom half (compare with the dashed line near the target). While an asymmetric profile is expected, the nature of the asymmetry is surprising. One might expect that the plasma would expand more along the magnetic field lines, as on the top half, and be more confined when expanding against the field lines, as on the bottom half. However, as we can see by comparing the diagram in Fig. 14a with Fig. 14b this is the opposite of what is observed experi- mentally. We can rule out the contribution of the laser ponderomotive pressure on the top side of the image, because the laser pulse duration is only $0.6 \mathrm{~ns}$ long and is thus long absent at 19 ns observed in the image. Further study is required to understand this asymmetric phenomena near the target. At distances further from the target (> $4 \mathrm{~mm}$ ) at $19 \mathrm{~ns}$ the plasma has tilted upwards, so that this portion of the flow begins to follow the magnetic field lines.

The most dramatic feature of the $45^{\circ}$ tilted field is shown at $60 \mathrm{~ns}$ in the Fig. 14c frame. Here there is no observation of a collimated jet, which is completely different than all other cases when the field was un-tilted. This suggests that there is a critical alignment angle between the plasma flow and magnetic field that dictates whether collimation is possible. This finding is critically important in understanding the viability of jet formation in astrophysical cases, because it places a constraint upon the orientation of ejected winds with the magnetic field. This encourages continued study to understand at which angles it is possible to create a jet and how this compares with the alignment of jets to magnetic fields in astronomical observations.

As the collimation of the jet is directly related to the formation of the poloidal magnetic nozzle, we expect that the asymmetry of the field somehow disrupts the formation of the PMN. This is substantiated by the fact that the radial profile of the plasma near the target is considerably different in the $45^{\circ}$ case; it is asymmetric and there is not a strong density increase at the edges that would indicate the formation of a shock.

\section{Summary}

The evolution of a narrow laser-generated jet collimated via a poloidal (axial) magnetic field[1, 2] was investigated over long spatial and temporal scales, by observing the electron density, electron temperature and optical emission. These diagnostic were used to characterize the plasma parameters and used to identify relevant non-dimensional scaling parameters that will aid in placing these experiments in an astrophysical context and suggesting new experimental studies. We introduced the terminology of a poloidal magnetic nozzle (PMN) to clarify that, as observed experimentally, the collimation of the flow is due mostly to the formation and structure at its base, and that a magnetic field is not necessary to keep the jet collimated as it extends over long distances.

We have found that varying the laser intensity and target material makes quantitative differences in the plasma collimation and jet formation, but that the general dynamics are very similar. On the other hand, variation of the magnetic field orientation by $45^{\circ}$ inhibits the formation of the PMN and thus does not form a jet.

The authors acknowledge the support of the LULI technical teams in the execution of this work. This work was 
supported by ANR Blanc Grant no 12-BS09-025-01 SILAMPA. We thank the Dresden High Magnetic Field Laboratory at Helmholtz-Zentrum Dresden-Rossendorf for the development of the pulsed power generator. We thank B. Albertazzi and M. Nakatsutsumi for their prior work in laying the groundwork for the experimental platform. This work was partly done within the LABEX PlasPar project and supported by Grant No. 11-IDEX-0004-02 from Agence Nationale de la Recherche. This work was supported in part by the Ministry of Education and Science of the Russian Federation under Contract No. 14.Z50.31. S. Pikuz acknowledges the support of RFBR foundation in the frame of projects \#14-29-06099 and \#15-32-21121. O.W. would like to acknowledge the DFG Programmes GRK 1203 and SFB/TR18. This work was performed under the auspices of the U.S. Department of Energy by Lawrence Livermore National Laboratory under Contract DE-AC52-07NA27344.

[1] A. Ciardi, T. Vinci, J. Fuchs, B. Albertazzi, C. Riconda, H. Pépin, O. Portugall, Astrophysics of magnetically collimated jets generated from laser-produced plasmas, Phys. Rev. Lett. 110 (2013) 025002. doi:10.1103/PhysRevLett.110.025002.

[2] B. Albertazzi, A. Ciardi, M. Nakatsutsumi, T. Vinci, J. Béard, R. Bonito, J. Billette, M. Borghesi, Z. Burkley, S. Chen, et al., Laboratory formation of a scaled protostellar jet by coaligned poloidal magnetic field, Science 346 (6207) (2014) 325-328.

[3] M.-E. Manuel, C. Kuranz, A. Rasmus, S. Klein, M. MacDonald, M. Trantham, J. Fein, P. Belancourt, R. Young, P. Keiter, et al., Experimental results from magnetized-jet experiments executed at the jupiter laser facility, High Energy Density Phys. 17 (2015) 52.

[4] A. C. Raga, L. Binette, J. Canto, N. Calvet, Stellar jets with intrinsically variable sources, Astrophys. J. 364 (1990) 601.

[5] A. Raga, S. Cabrit, C. Dougados, C. Lavalley, A precessing, variable velocity jet model for dg tauri, Astron. Astrophys. 367 (3) (2001) 959.

[6] S. Matt, R. Winglee, K.-H. Böhm, Collimation of a central wind by a disc-associated magnetic field, Mon. Not. R. Astron. Soc. 345 (2) (2003) 660-670.

[7] J. Kwan, E. Tademaru, Jets from t tauri stars-spectroscopic evidence and collimation mechanism, Astrophys. J. 332 (1988) L41-L44.

[8] H. C. Spruit, T. Foglizzo, R. Stehle, Collimation of magnetically driven jets from accretion discs, Mon. Not. R. Astron. Soc. 288 (2) (1997) 333-342.

[9] F. Favata, C. V. M. Fridlund, G. Micela, S. Sciortino, A. A. Kaas, Discovery of x-ray emission from the protostellar jet 11551 irs5 (hh 154), Astron. Astrophys. 386 (1) (2002) 204-210.

[10] J. Bally, E. Feigelson, B. Reipurth, X-rays from the vicinity of the protostar 11551 irs 5: Reflection or fast shocks?, Astrophys. J. 584 (2) (2003) 843.

[11] R. Bonito, S. Orlando, M. Miceli, G. Peres, G. Micela, F. Favata, X-ray emission from protostellar jet hh 154: The first evidence of a diamond shock?, Astrophys. J. 737 (2) (2011) 54.

[12] D. D. Ryutov, R. P. Drake, B. A. Remington, Criteria for scaled laboratory simulations of astrophysical mhd phenomena, Astrophys. J. Suppl. Ser. 127 (2) (2000) 465.

[13] D. Ryutov, R. P. Drake, J. Kane, E. Liang, B. A. Remington, W. M. Wood-Vasey, Similarity criteria for the laboratory simulation of supernova hydrodynamics, Astrophys. J. 518 (2) (1999) 821.

[14] A. Frank, T. Ray, S. Cabrit, P. Hartigan, H. Arce, F. Bacciotti, J. Bally, M. Benisty, J. Eislöffel, M. Güdel, S. Lebedev, B. Nisini, A. Raga, Jets and outflows from star to cloud: Observations confront theory, Protostars and Planets VI 1 (2014) 451-474.
[15] R. D. Blandford, D. G. Payne, Hydromagnetic flows from accretion discs and the production of radio jets, Mon. Not. R. Astron. Soc. 199 (1982) 883-903.

[16] T. Matsakos, S. Massaglia, E. Trussoni, K. Tsinganos, N. Vlahakis, C. Sauty, A. Mignone, Two-component jet simulations-ii. combining analytical disk and stellar mhd outflow solutions, Astron. Astrophys. 502 (1) (2009) 217-229.

[17] S. Lebedev, A. Ciardi, D. Ampleford, S. Bland, S. Bott, J. Chittenden, G. Hall, J. Rapley, C. Jennings, A. Frank, et al., Magnetic tower outflows from a radial wire array z-pinch, Mon. Not. R. Astron. Soc. 361 (1) (2005) 97-108.

[18] D. J. Ampleford, S. V. Lebedev, A. Ciardi, S. N. Bland, S. C. Bott, G. N. Hall, N. Naz, C. A. Jennings, M. Sherlock, J. P. - Chittenden, et al., Supersonic radiatively cooled rotating flows and jets in the laboratory, Phys. Rev. Lett. 100 (3) (2008) 035001.

[19] A. Ciardi, S. V. Lebedev, A. Frank, F. Suzuki-Vidal, G. N. Hall, S. N. Bland, A. Harvey-Thompson, E. G. Blackman, M. Camenzind, Episodic magnetic bubbles and jets: astrophysical implications from laboratory experiments, Astrophys. J. 691 (2) (2009) L147.

[20] E. W. Sucov, J. L. Pack, A. V. Phelps, A. G. Engelhardt, Plasma production by a high-power q-switched laser, Phys. Fluids 10 (9) (1967) 2035-2048.

[21] J. Bruneteau, E. Fabre, H. Lamain, P. Vasseur, Experimental investigation of the production and containment of a laserproduced plasma, Phys. Fluids 13 (7) (1970) 1795-1801.

[22] T. A. Peyser, C. K. Manka, B. H. Ripin, G. Ganguli, Electronion hybrid instability in laser-produced plasma expansions across magnetic fields, Phys. Fluids B 4 (8) (1992) 2448-2458.

[23] A. N. Mostovych, B. H. Ripin, J. A. Stamper, Laser produced plasma jets: collimation and instability in strong transverse magnetic fields, Phys. Rev. Lett. 62 (24) (1989) 2837.

[24] C. Plechaty, R. Presura, A. A. Esaulov, Focusing of an explosive plasma expansion in a transverse magnetic field, Phys. Rev. Lett. 111 (18) (2013) 185002.

[25] B. H. Ripin, J. D. Huba, E. A. McLean, C. K. Manka, T. Peyser, H. R. Burris, J. Grun, Sub-alfvénic plasma expansion, Phys. Fluids B 5 (10) (1993) 3491-3506.

[26] Y. P. Zakharov, V. Antonov, E. L. Boyarintsev, A. Melekhov, V. Posukh, I. Shaikhislamov, V. Pickalov, Role of the hall flute instability in the interaction of laser and space plasmas with a magnetic field, Plasma Phys. Rep. 32 (3) (2006) 183-204.

[27] O. V. Gotchev, J. P. Knauer, P. Y. Chang, N. W. Jang, M. J. Shoup III, D. D. Meyerhofer, R. Betti, Seeding magnetic fields for laser-driven flux compression in high-energy-density plasmas, Rev. Sci. Instrum. 80 (4) (2009) 043504.

[28] O. Gotchev, P. Chang, J. Knauer, D. Meyerhofer, O. Polomarov, J. Frenje, C. Li, M.-E. Manuel, R. Petrasso, J. Rygg, F. H. Séguin, R. Betti, Laser-driven magnetic-flux compression in high-energy-density plasmas, Phys. Rev. Lett. 103 (21) (2009) 215004.

[29] P. Y. Chang, G. Fiksel, M. Hohenberger, J. P. Knauer, R. Betti, F. J. Marshall, D. D. Meyerhofer, F. H. Séguin, R. D. Petrasso, Fusion yield enhancement in magnetized laser-driven implosions, Phys. Rev. Lett. 107 (3) (2011) 035006.

[30] S. Fujioka, Z. Zhang, K. Ishihara, K. Shigemori, Y. Hironaka, T. Johzaki, A. Sunahara, N. Yamamoto, H. Nakashima, T. Watanabe, H. Shiraga, H. Nishimura, H. Azechi, Kilotesla magnetic field due to a capacitor-coil target driven by high power laser, Scientific reports 3.

[31] B. Albertazzi, J. Béard, A. Ciardi, T. Vinci, J. Albrecht, J. Billette, T. Burris-Mog, S. Chen, D. Da Silva, S. Dittrich, et al., Production of large volume, strongly magnetized laser-produced plasmas by use of pulsed external magnetic fields, Rev. Sci. Instrum. 84 (4) (2013) 043505.

[32] D. P. Higginson, P. Korneev, J. Béard, S. N. Chen, E. d'Humières, H. Pépin, S. Pikuz, B. Pollock, R. Riquier, V. Tikhonchuk, J. Fuchs, A novel platform to study magnetized high-velocity collisionless shocks, High Energy Density Physics 17 (2015) 190-197. 
[33] A. Faenov, V. Dyakin, A. Magunov, T. Pikuz, I. Skobelev, S. Pikuz, A. Kasperczyk, T. Pisarczyk, J. Wolowski, E. Zielinska, Using x-ray spectroheliograph technique for investigations of laser-produced plasma under interaction with strong magnetic field, Physica Scripta 53 (5) (1996) 591.

[34] B. B. Pollock, D. H. Froula, G. R. Tynan, L. Divol, D. Price, R. Costa, F. Yepiz, S. Fulkerson, F. Mangini, S. H. Glenzer, Multicentimeter long high density magnetic plasmas for optical guidinga), Rev. Sci. Instrum. 79 (10) (2008) 10F550.

[35] L. Lancia, B. Albertazzi, C. Boniface, A. Grisollet, R. Riquier, F. Chaland, K.-C. Le Thanh, P. Mellor, P. Antici, S. Buffechoux, S. Chen, D. Doria, M. Nakatsutsumi, C. Peth, M. Swantusch, M. Stardubtsev, L. Palumbo, M. Borghesi, O. Willi, H. Ppin, J. Fuchs, Topology of megagauss magnetic fields and of heat-carrying electrons produced in a high-power laser-solid interaction, Phys. Rev. Lett. 113 (23) (2014) 235001.

[36] L. Gao, P. Nilson, I. Igumenshchev, M. Haines, D. Froula, R. Betti, D. Meyerhofer, Precision mapping of laser-driven magnetic fields and their evolution in high-energy-density plasmas, Phys. Rev. Lett. 114 (21) (2015) 215003.

[37] B. A. Remington, R. P. Drake, D. D. Ryutov, Experimental astrophysics with high power lasers and z pinches, Rev. Mod. Phys. 78 (3) (2006) 755.

[38] S. C. Hsu, P. M. Bellan, Experimental identification of the kink instability as a poloidal flux amplification mechanism for coaxial gun spheromak formation, Phys. Rev. Lett. 90 (21) (2003) 215002.

[39] P. M. Bellan, S. You, S. C. Hsu, Simulating astrophysical jets in laboratory experiments, Astrophysics and Space Science 298 (12) (2005) 203-209.

[40] D. Farley, K. Estabrook, S. Glendinning, S. Glenzer, B. Remington, K. Shigemori, J. Stone, R. Wallace, G. Zimmerman, J. Harte, Radiative jet experiments of astrophysical interest using intense lasers, Phys. Rev. Lett. 83 (10) (1999) 1982.

[41] J. M. Foster, B. H. Wilde, P. A. Rosen, T. S. Perry, M. Fell, M. J. Edwards, B. F. Lasinski, R. E. Turner, M. L. Gittings, Supersonic jet and shock interactions, Phys. Plasmas 9 (5) (2002) 2251-2263.

[42] P. Nicolai, C. Stenz, V. Tikhonchuk, A. Kasperczuk, T. Pisarczyk, L. Juha, E. Krousky, K. Masek, M. Pfeifer, K. Rohlena, et al., Experimental evidence of multimaterial jet formation with lasers, Phys. Plasmas 17 (11) (2010) 112903

[43] B. Loupias, M. Koenig, E. Falize, S. Bouquet, N. Ozaki, A. Benuzzi-Mounaix, T. Vinci, C. Michaut, M. R. Le Goahec, W. Nazarov, et al., Supersonic-jet experiments using a highenergy laser, Phys. Rev. Lett. 99 (26) (2007) 265001.

[44] R. Yurchak, A. Ravasio, A. Pelka, S. Pikuz Jr, E. Falize, T. Vinci, M. Koenig, B. Loupias, A. Benuzzi-Mounaix, M. Fatenejad, P. Tzeferacos, D. Lamb, E. Blackman, Experimental demonstration of an inertial collimation mechanism in nested outflows, Phys. Rev. Lett. 112 (15) (2014) 155001.

[45] J. Zou, C. Blanc, P. Audebert, S. Janicot, A. Sautivet, L. Martin, C. Sauteret, J. Paillard, S. Jacquemot, F. Amiranoff, Recent progress on luli high power laser facilities, J. Phys.: Conf. Ser. 112 (3) (2008) 032021.

[46] D. Strickland, G. Mourou, Compression of amplified chirped optical pulses, Opt. Commun. 55 (6) (1985) 447-449.

[47] Y. Kato, K. Mima, N. Miyanaga, S. Arinaga, Y. Kitagawa, M. Nakatsuka, C. Yamanaka, Random phasing of high-power lasers for uniform target acceleration and plasma-instability suppression, Phys. Rev. Lett. 53 (11) (1984) 1057.

[48] J. Béard, F. Debray, The french high magnetic field facility, J. Low Temp. Phys. 170 (5-6) (2013) 541-552.

[49] J. Béard, J. Billette, P. Frings, M. Suleiman, F. Lecouturier, Special coils development at the national high magnetic field laboratory in toulouse, J. Low Temp. Phys. 170 (5-6) (2013) $442-446$

[50] T. Vinci, A. Flacco, Neutrino (2014). URL https://github.com/aflux/neutrino

[51] K. Bockasten, Transformation of observed radiances into radial distribution of the emission of a plasma*, J. Opt. Soc. Am.
51 (9) (1961) 943.

[52] J. Albrecht, J.-R. Marquès, personal communication.

[53] S. N. Ryazantsev, I. Y. Skobelev, A. Y. Faenov, T. A. Pikuz, A. N. Grum-Grzhimailo, S. A. Pikuz, JETP Letters 102 (2015) 817.

[54] S. N. Ryazantsev, I. Y. Skobelev, A. Y. Faenov, T. A. Pikuz, A. N. Grum-Grzhimailo, S. A. Pikuz, submitted to Phys. Plasmas.

[55] H. Chung, M. H. Chen, W. L. Morgan, Y. Ralchenko, R. W. Lee, Flychk: Generalized population kinetics and spectral model for rapid spectroscopic analysis for all elements, High Energy Density Phys. 1 (1) (2005) 3-12.

[56] S. I. Braginskii, Transport processes in a plasma, Reviews of plasma physics 1 (1965) 205.

[57] M. D. Rosen, R. H. Price, E. M. Campbell, D. W. Phillion, K. G. Estabrook, B. F. Lasinski, J. M. Auerbach, S. P. Obenschain, E. A. McLean, R. R. Whitlock, et al., Analysis of laserplasma coupling and hydrodynamic phenomena in long-pulse, long-scale-length plasmas, Phys. Rev. A 36 (1) (1987) 247.

[58] H. Puell, Heating of laser produced plasmas generated at plane solid targets i. theory, Zeitschrift für Naturforschung A 25 (12) (1970) 1807-1815.

[59] R. P. Drake, G. Gregori, Design considerations for unmagnetized collisionless-shock measurements in homologous flows, Astrophys. J. 749 (2) (2012) 171.

[60] D. Ryutov, N. Kugland, H.-S. Park, S. Pollaine, B. Remington, J. Ross, Collisional current drive in two interpenetrating plasma jets, Phys. Plasmas 18 (10) (2011) 104504.

[61] D. D. Ryutov, N. L. Kugland, H. Park, C. Plechaty, B. A. Remington, J. S. Ross, Intra-jet shocks in two counter-streaming, weakly collisional plasma jets, Phys. Plasmas 19 (7) (2012) 074501 .

[62] D. D. Ryutov, N. L. Kugland, M. Levy, C. Plechaty, J. S. Ross, H. Park, Magnetic field advection in two interpenetrating plasma streams, Phys. Plasmas 20 (3) (2013) 032703.

[63] H. Hornung, Regular and mach reflection of shock waves, Annu. Rev. Fluid Mech. 18 (1986) 33-58. 